\title{
Patent Licensing, Entry and the Incentive to Innovate
}

\author{
Yair Tauman ${ }^{* 1}$ and Chang Zhao ${ }^{\dagger 2}$ \\ ${ }^{1}$ Stony Brook University and The Interdisciplinary Center, Herzliya \\ ${ }^{2}$ Stony Brook University
}

\begin{abstract}
We analyze the economic impact of process innovations where the innovator auctions off licenses to both potential entrants and incumbent firms. It is shown that opening the market to entrant licensees, the incentive to innovate is maximized in a monopoly market as was envisioned by Schumpeter $(1942)$. This is in contrast to previous literature on licensing of process innovations where entry is excluded. There the incentive to innovate is maximized in oligopoly market if licenses are sold by auction (Sen and Tauman (2007)) or in competitive market if licenses are sold by royalty (Arrow (1962)). The post-innovation market structure, the diffusion of the innovation and the social welfare are analyzed and compared with the case where entry is excluded.
\end{abstract}

Keywords. Innovation, Patent Licensing, Entry, Incentive to Innovate

\footnotetext{
${ }^{*}$ Corresponding Author. Interdisciplinary Center (IDC) Herzliya, Israel. Tel: +972 99527984 . Email address: amty21@gmail.com

${ }^{\dagger}$ Present Address: School of Mathematical Sciences, Tel Aviv University. Email address: zhaochangtd@gmail.com
} 


\section{Introduction}

The analysis of optimal licensing strategies of an innovator, the post innovation market structure as well as the incentive to innovate has been extensively studied in the literature, starting with Katz and Shapiro (1985), Katz (1986), Kamien and Tauman (1984), Kamien and Tauman (1986), Kamien, Oren, and Tauman (1992). A review of the first decade results on this topic is Kamien (1992). The literature on the optimal market structure which provides the highest incentive to innovate starts with Arrow (1962) showing that the revenue of an innovator who sells licenses by means of a per-unit royalty is maximized in a competitive market. Kamien and Tauman (1986) and the extended analysis in Sen and Tauman (2007) show that the revenue of an innovator who sells licenses by either an upfront fee determined by an auction or by a per-unit royalty (or by a combination of the two) is maximized in an oligopoly market of a size which depends on the magnitude of innovation, demand intensity and the marginal cost of production. In these papers, as well as most other papers on optimal licensing of new innovations, it is assumed that incumbent firms are the only potential licensees.

This paper analyzes the economic impact of process innovations where the buyers are not symmetric. Namely, the innovator can sell licenses to both potential entrants and incumbent firms. Licenses in our model are sold by auction aiming to maximize the revenue of the innovator. The post-innovation market structure, the diffusion of the innovation and the incentive to innovate are compared with the case where licenses are sold only to incumbent firms and not to entrants.

In contrast to the literature on licensing of process innovation to incumbent firms only, it is shown, quite surprisingly, that opening the Cournot market to entrant licensees, the incentive to innovate is maximized in a monopoly market rather than oligopoly or competitive markets and this is true for drastic as well as non-drastic innovations. The total number of licensees is no longer constrained by the number of incumbent firms and each licensee's willingness to pay for a license is higher the smaller is the pre-innovation market size. This result is 
consistent with the observation of Schumpeter (1942) that monopolistic industries, those in which individual firms have a measure of control over their products price, provide a more hospitable atmosphere for innovation than purely competitive ones. This is also in line with Chen and Schwartz (2013), who show that the gain of a innovator from the exclusive use of a product innovation can be larger in a monopoly market than in a perfectly competitive one.

It is further shown that the innovator sells licenses to entrants only if he also sells licenses to all incumbent firms. Although entrants are willing to pay for a license typically more than incumbent firms, the competition effect on the revenue of the innovator dominates his revenue from additional entrant. Furthermore, the post-innovation market size is larger the smaller is the magnitude of innovation. Namely less significant innovations diffuse more. To clarify this point notice that the (negative) competition effect of an additional licensee on the innovator's revenue is increasing in the magnitude of the innovation and as a result the innovator is more reluctant to issue a large number of licenses for more significant innovations. Consequently, for innovations with sufficiently high magnitude the innovator chooses to sell licenses only to incumbent firms and not to entrants. In this case the diffusion of the innovation is the same as in the case where entry is excluded. For less significant innovations the innovator sells licenses to some entrants and to all incumbent firms. In this case opening the market to entrants has positive effect on social welfare and it yields the innovator a higher revenue, compare to the case where entry is excluded. The marginal effect of entry on the innovator's revenue as well as on the social welfare is higher, the less significant is the magnitude of innovation.

We are aware of only few papers which deals with the licensing of innovations to both incumbent firms and potential entrants. The one closest to this paper is Hoppe, Jehiel, and Moldovanu (2006), (HJM here after). In HJM the innovator sells licenses through a uniform auction (UA). The innovator in UA chooses the number $k$ of licenses to sell. The auction welcomes bids from both incumbent firms and entrants. Each one of the $\mathrm{k}$ highest 
bidders (whether incumbent firm or entrant) obtains a license and all licensees pay the same amount, the $(k+1)$ th highest bid. Externality plays an important role in UA. Incumbent firms and entrants have different willingness to pay for a license and both of them depend not only on the number of licensees but also on the distribution of entrants and incumbent licensees. The problem however with UA is that it has not only multiple equilibrium points but also multiple equilibrium payoffs for the innovator, even if weakly dominated strategies are excluded. HJM in a general setup mostly deals with the sale of an exclusive license as well as some special cases involving multiple licenses and focuses on whether entrants can be winners of licenses. Our paper while provides a general analysis of the optimal licensing strategy of the innovation it is done in a specific set-up: Cournot oligopoly market, linear demand and a constant per-unit cost. We show that for any number of licenses, $k \geq 1$, every partition $\left(k_{1}, k_{2}\right)$ of $k\left(k_{1}+k_{2}=k\right)$ can be supported as an equilibrium outcome, where $k_{1}$ is the number of incumbent licensees and $k_{2}$ is the number of entrant licensees. The innovator only controls $k$ and has no control over the partition of $k$ into incumbent and entrant licensees, making it difficult if not impossible to predict the outcome of UA. In particular, it is not clear what would be the innovator's choice of $k$. To have some UA benchmark we compute the highest equilibrium payoff of the innovator in UA and compare it with the equilibrium outcomes of two alternative types of auction. The first one is NUA (non-uniform auction). The second one is SUA (semi-uniform auction) who has a weaker asymmetry than NUA.

The innovator in NUA chooses in addition to $k$, the exact partition $\left(k_{1}, k_{2}\right)$ of $k$. The winners of the auctions are the $k_{1}$ highest incumbent bidders and the $k_{2}$ highest entrant bidders (ties are resolved at random). Each incumbent licensee pays the $\left(k_{1}+1\right)$ th highest bid among the incumbents' bids while each entrant licensee pays the $\left(k_{2}+1\right)$ th highest bid among the entrants' bids! In contrast to UA, externalities do not play a role in NUA since

\footnotetext{
${ }^{1}$ We will obtain the same equilibrium outcome if the license fee incumbents (entrants) pay is the $k_{1}$-th $\left(k_{2}\right.$-th) highest bid among the incumbent (entrant) bids. Setting the license fee to be the $\left(k_{1}+1\right)$ th highest bid among incumbent bids guarantee that bidding truthfully (the true willingness to pay) is a weakly dominant strategy of every incumbent firm (similar for entrants).
} 
the post-innovation market structure is determined by $\left(k_{1}, k_{2}\right)$ regardless of the bids. Given $\left(k_{1}, k_{2}\right)$, the equilibrium outcome in undominated strategies is uniquely determined.

In the first glance it seems that the ability to choose $\left(k_{1}, k_{2}\right)$ and to differentiate the license fee of entrants from incumbent firms should yield the innovator in NUA a higher payoff than in UA. But this may not be the case. On one hand every entrant licensee pays in NUA her entire profit (assuming zero entrant's opportunity cost) while in UA it is (like any incumbent licensee) only the incremental profit of an incumbent licensee. However, on the other hand an incumbent licensee is willing to pay more in UA if he takes the place of an entrant licensee and hence limit entry. In contrast every incumbent licensee in NUA takes the place of another incumbent firm and thus does not change the number of active firms. It is shown that the innovator's highest possible equilibrium payoff in UA is higher than his unique equilibrium payoff in NUA if and only if the magnitude of the innovation is sufficiently high.

In the semi-uniform auction (SUA), the innovator, like in NUA, chooses both $k$ and the exact partition $\left(k_{1}, k_{2}\right)$ of $k$. The winners of the auctions are the $k_{1}$ highest incumbent bidders and the $k_{2}$ highest entrant bidders (ties are resolved at random). But unlike NUA, the license fee is the same across all licensees. To ensure the participation of incumbent firms in SUA the license fee is set to be the $k$-th highest bid ${ }^{2}$.

It is shown that irrespective of the market size and the magnitude of innovation the revenue the innovator extracts in SUA does not exceed his revenue in NUA. Like in NUA, when selling licenses by SUA, a monopoly market provides the highest incentive to innovate. For relatively significant innovation the total number of licenses the innovator sells is decreasing in the magnitude of the innovation and the innovator sells licenses to entrants only if he also sells licenses to all incumbent firms. In contrast to NUA, for less significant innovations the innovator in SUA sells licenses only to new entrants and not to incumbent firms. The reason

\footnotetext{
${ }^{2}$ If (as in UA) every licensee pays the highest losing bid, it may happen that the bids of all incumbent licensees fall below the $(k+1)$ th highest bid. This is the case if the $(k+1)$ th highest bid is submitted by an entrant who bids her entire industry profit. Such bid exceeds the willingness to pay of incumbent firms and incumbents are best off not participating in this auction.
} 
is his ability to extract the entire industry profit of every entrant licensee, as opposed to the case where he sells some licenses also to incumbent firms. In the latter case the license fee an entrant pays is equal to the willingness to pay of an incumbent licensee which decreases to zero as the magnitude of innovation decreases to zero. In contrast, the innovator in NUA can discriminate entrant licensees and he extracts their entire industry profit, whether or not he sells licenses to incumbent firms. Therefore even for less significant innovations in NUA the innovator sells licenses to both new entrants and incumbent firms. Consequently, for less significant innovations, NUA results in a higher diffusion of technology. We conclude that for less significant innovations the ability to price discriminate new entrant licensees has positive effect not only on the innovator's revenue but also on social welfare, as compare to SUA.

Finally, a recent paper studying the innovator's optimal licensing strategy in the presence of potential entry is Tauman, Weiss, and Zhao ("Bargaining in Patent Licensing with Inefficient Outcomes"). This paper consider a process innovation in a monopoly industry. The innovator bargains with the monopolist over the IP of the innovation. To induce the monopolist to pay a higher fee, the innovator before approaching the monopolist can (and sometimes does) sell a few licenses to new entrants. On one hand selling licenses to entrants reduces the total industry profit (the "cake" to be allocated in bargaining) but serves as a credible threat on the monopolist to sell even more licenses to new entrants if the bargaining fails.

\section{The Model}

Consider an industry with a set $N=\{1, \ldots, n\}$ of incumbent firms who produce one product with marginal cost $c>0$. Potential entrants are unable to enter the market either because of high fixed cost or since the current technology is protected by patent. An outside innovator comes along with an innovation which eliminates the fixed cost and reduces the 
constant per unit cost from $c$ to $c-\epsilon, 0<\epsilon \leq c$. The $\AA^{3}$ number of potential entrants is assumed to be sufficiently large and it exceeds the optimal number of licenses sold by the innovator.

The inverse demand function is linear, $p=\max (a-Q, 0)$. Denote by $\pi_{1}\left(m_{0}, m_{1}\right)$ and $\pi_{0}\left(m_{0}, m_{1}\right)$ the Cournot profit of a licensee and a non-licensee, respectively, when there are $m_{0}$ firms producing at a unit $\operatorname{cost} c$ and $m_{1}$ firms producing at a unit $\operatorname{cost} c-\epsilon$. It can be verified that

$$
\begin{gathered}
\pi_{0}\left(m_{0}, m_{1}\right)= \begin{cases}\left(\frac{(a-c)-\epsilon m_{1}}{m_{0}+m_{1}+1}\right)^{2} & \text { if } m_{1} \leq \frac{a-c}{\epsilon} \\
0 & \text { if } m_{1}>\frac{a-c}{\epsilon}\end{cases} \\
\pi_{1}\left(m_{0}, m_{1}\right)= \begin{cases}\left(\frac{(a-c)+\left(m_{0}+1\right) \epsilon}{m_{0}+m_{1}+1}\right)^{2} & \text { if } m_{1} \leq \frac{a-c}{\epsilon} \\
\left(\frac{(a-c)+\epsilon}{m_{1}+1}\right)^{2} & \text { if } m_{1}>\frac{a-c}{\epsilon}\end{cases}
\end{gathered}
$$

Without loss of generality we normalize $a-c$, the quantity demanded at the price $c$, to be 1. We make the following assumption throughout the paper.

Assumption 1. Training and installing the new technology is costly and paid only by the innovator. This cost is sufficiently small and has no effect on the optimal number of licenses the innovator sells. The training cost is smaller for incumbent licensees than entrant licensees.

This assumption simplifies the tie breaking rule. If a tie involves both entrants and incumbent firms, the innovator prefers to sell a license to an incumbent firm. If a tie involves only one type of bidders, the tie is resolved at random.

In UA the players are engaged in a three-stage game, $G_{u}$. In the first stage the innovator chooses and announces the number $k$ of licenses to be auctioned off, to both incumbent firms and new entrants. In the second stage the licenses are allocated to the winners of a uniform auction where each one of the $k$ highest bidders obtains a license and pays the $(k+1)$ th

\footnotetext{
${ }^{3}$ In principle even $\epsilon \leq 0$ may be valuable, and entrants may be willing to pay for inefficient technology if it allows them a profitable entry. We confine in this paper to $\epsilon>0$.
} 
highest bid. In the third and last stage the firms (incumbents and entrant licensees) compete à la Cournot. Let $G_{u}(k)$ be the subgame of $G_{u}$ which starts after the announcement of $k$.

Let $G_{n u}$ be the game associated with NUA. In the first stage the innovator chooses and announces $\left(k_{1}, k_{2}\right)$, where $0 \leq k_{1} \leq n-1$ and $k_{2} \geq 0$ are the number of licenses he auctions off to incumbent firms and entrants, respectively. Let $G_{n u}\left(k_{1}, k_{2}\right)$ be the subgame of $G_{n u}$ which starts in the second stage of $G_{n u}$. In $G_{n u}\left(k_{1}, k_{2}\right)$, licenses are sold through a non-uniform auction. Each of the $k_{1}$ highest incumbent bidders obtains a license and pays the $\left(k_{1}+1\right)$ th highest bid among the incumbents' bids. Similarly, each of the $k_{2}$ highest entrant bidders obtains a license and pays the $\left(k_{2}+1\right)$ th highest bid among the entrants' bids. In the third stage the firms in the industry (licensees and non-licensees) engage in Cournot competition. Note that the auction is not well define for $k_{1}=n$. Thus we limit $k_{1}$ to $n-1$.

In $G_{n u}\left(k_{1}, k_{2}\right)$, the value of a license is uniquely determined for each bidder. This is not the case in UA where the value of a license typically depends on the distribution of incumbent and entrant licensees. Note that bidders do not usually have dominant strategies in UA.

Proposition 1. Suppose bidders do not use dominated strategies. (i) If the innovator auctions off a total of $\frac{1}{\epsilon}$ licenses (using either UA or NUA), then the Cournot price is c, the pre-innovation marginal cost, and every non-licensee firm is driven out of the market. Each licensee pays his entire profit and the innovator obtains the total industry profit. (ii) It is never optimal for the innovator in both UA and NUA to auction off more than $\frac{1}{\epsilon}$ licenses.

It will be shown (see Proposition 3 and Proposition 5 , below) that for $\epsilon>\frac{2}{n+1}$ the optimal number of licenses for the innovator is $k=\frac{1}{\epsilon}$ in both UA and NUA .

Proof. Part (i) is a straight forward consequence of (1). Part (i) asserts that when $k=\frac{1}{\epsilon}$ only licensees are active firms in the market, and this is obviously true for all $k \geq \frac{1}{\epsilon}$. Since the total industry profit is decreasing in $k$ for $k \geq \frac{1}{\epsilon}$, part (ii) follows.

By Proposition 1, without loss of generality we only consider the case where in both UA 
and NUA the total number $k$ of licenses does not exceed $\frac{1}{\epsilon}$. In case $\epsilon \geq 1$ (drastic innovation) even if the innovator sells an exclusive license, every non-licensee firm is driven out of the market and the innovator extracts the monopoly profit under the new technology. It is left to analyze only the non-drastic innovation case, namely $\epsilon<1$.

\subsection{Uniform Auction}

Consider the subgame $G_{u}(k)$ of $G_{u}$, for $1 \leq k \leq \frac{1}{\epsilon}$. Suppose $\left(k_{1}, k_{2}\right)$ is an equilibrium outcome of $G_{u}(k)$, where $k_{1}, 0 \leq k_{1} \leq n$, is the number of incumbent licensees and $k_{2}=k-k_{1}$ is the number of entrant licensees. Let $b_{(i)}$ be the $i$ th highest bid in UA $\left(b_{(i)}=b_{(i+1)}\right.$ if more than one bidder bids $\left.b_{(i)}\right)$.

The willingness to pay of an incumbent firm, $i$, for a license is the difference between his profit $\pi_{1}\left(n-k_{1}, k\right)$ as a licensee and his profit as a non-licensee in case he drops out. The latter depends on the type of licensee replacing $i$. If it is an entrant, the total number of firms increases by 1 and the willingness to pay of $i$ is

$$
w_{i h}^{k}\left(k_{1}\right)=\pi_{1}\left(n-k_{1}, k\right)-\pi_{0}\left(n-k_{1}+1, k\right)
$$

If $i$ is replaced by another incumbent firm, his willingness to pay is

$$
w_{i l}^{k}\left(k_{1}\right)=\pi_{1}\left(n-k_{1}, k\right)-\pi_{0}\left(n-k_{1}, k\right) .
$$

Note that $w_{i h}^{k}$ can be regarded as an incumbent's willingness to pay for limiting entry and using the superior technology.

The willingness to pay of an entrant for a license is simply her Cournot profit,

$$
w_{e}^{k}\left(k_{1}\right)=\pi_{1}\left(n-k_{1}, k\right)
$$

By Proposition 11, for any $k_{1}, 0 \leq k_{1} \leq \min (k, n)$, if $k \geq \frac{1}{\epsilon} w_{e}^{k}\left(k_{1}\right)=w_{i h}^{k}\left(k_{1}\right)=w_{i l}^{k}\left(k_{1}\right)$. If 
$k<\frac{1}{\epsilon}$ then $\pi_{0}\left(n-k_{1}, k\right)>\pi_{0}\left(n-k_{1}+1, k\right)>0$ and $w_{e}^{k}\left(k_{1}\right)>w_{i h}^{k}\left(k_{1}\right)>w_{i l}^{k}\left(k_{1}\right)$. Namely for $k<\frac{1}{\epsilon}$ any entrant licensee is willing to pay for a license more than any incumbent licensee. Nevertheless it is still possible that in equilibrium some incumbent firm wins a license. To clarify this point observe that an entrant with no license who outbids an incumbent licensee not only increases the number of active firms by 1 but also increases the license fee from $b_{(k+1)}$ to $b_{(k)}$. This may reduce the profit of each licensee to a level below $b_{(k)}$, causing the deviant entrant a loss.

By Proposition 1, when the innovator chooses $k=\frac{1}{\epsilon}$ (or actually $k \geq \frac{1}{\epsilon}$ ), the willingness to pay of each bidder is independent of the distribution of licensees between entrants and incumbent firms and each bidder's willingness to pay in $G_{u}(k)$ is his Cournot profit. If, however, $k<\frac{1}{\epsilon}$, each bidder's willingness to pay depends in addition to $k$ on the distribution of winners. We next analyze the innovator's equilibrium payoff in this case.

Proposition 2. Let $1 \leq k<\frac{1}{\epsilon}$. Then (i) any $\left(k_{1}, k_{2}\right), 0 \leq k_{1} \leq n$ and $k_{2} \geq 0$ s.t. $k_{1}+k_{2}=k$, is an equilibrium outcome of $G_{u}(k)$. (ii) For $k_{1}=0, \pi$ is an equilibrium payoff of the innovator in $G_{u}(k)$ if and only if $\pi \in\left[0, k w_{e}^{k}(0)\right]$. (iii) For $1 \leq k_{1} \leq n, \pi$ is an equilibrium payoff of the innovator in $G_{u}(k)$ if and only if $\pi \in\left[0, k w_{i h}^{k}\left(k_{1}\right)\right]$.

Proof. (i) Let $k \geq 1$ and let $\left(k_{1}, k_{2}\right)$ s.t. $0 \leq k_{1} \leq n-1$ and $k_{2}=k-k_{1}$ (the case where $k_{1}=n$ will be dealt separately). Let us show that $\left(k_{1}, k_{2}\right)$ is an equilibrium outcome of $G_{u}(k)$. Denote $b=\pi_{1}\left(n-k_{1}-1, k\right)$ ( $b$ is well defined since $\left.k_{1} \leq n-1\right)$ and $\underline{b}=$ $\pi_{1}\left(n-k_{1}, k\right)-\pi_{0}\left(n-k_{1}+1, k\right)$. Suppose that exactly $k_{1}$ incumbent firms and $k_{2}$ entrants bid $b$ and only one entrant bids $\underline{b}$. All other incumbents or entrants bid below $\underline{b}$. Clearly $b_{(1)}=\ldots=b_{(k)}=b, b_{(k+1)}=\underline{b}$ and $\underline{b} \leq b$. We claim that these bid profile constitutes an equilibrium of $G_{u}(k)$. Any incumbent licensee, $i$, obtains

$$
\pi_{1}\left(n-k_{1}, k\right)-b_{(k+1)}=\pi_{0}\left(n-k_{1}+1, k\right) .
$$

If $i$ lowers his bid below $\underline{b}$ the entrant who bids $\underline{b}$ will replace $i$. As a result there will be 
$n-k_{1}+1$ firms producing with the inferior technology and $i$ will obtain $\pi_{0}\left(n-k_{1}+1, k\right)$, the same as his payoff as a licensee. Since the opportunity cost of any entrant is zero, an entrant licensee (when $k_{2} \geq 1$ ) has no incentive to lower her bid. Next let us show that a non-licensee (incumbent or entrant) can not benefit from outbidding a licensee. Suppose $j$ (incumbent or entrant) outbids a licensee $i$. Then he/she will increase the license fee from $\underline{b}$ to $b$. We claim that the industry profit of $j$ is at most $b$ and hence he has no incentive to become a licensee. Indeed, if both $j$ and $i$ are incumbent firms the industry profit of $j$ as a licensee will be $\pi_{1}\left(n-k_{1}, k\right)$ which is smaller than $b=\pi_{1}\left(n-k_{1}-1, k\right)$. If $j$ is an incumbent firm and $i$ is an entrant, the number of firms using the inferior technology will reduce to $n-k_{1}-1$. The gross profit of $j$ as a licensee will be $b$ and his payoff, net of the new license fee, is zero. If $j$ is an entrant, $j$ will obtain an industry profit of $\pi_{1}\left(n-k_{1}+1, k\right)<b$ if $i$ is an incumbent firm and $\pi_{1}\left(n-k_{1}, k\right)<b$ if $i$ is an entrant. In both cases $j$ 's net payoff is negative. To complete the proof of part (i) suppose that $k_{1}=n$ and hence $k_{2}=k-n$. Suppose every incumbent firm and exactly $k_{2}$ entrants bid $b=\pi_{1}(0, k)$, one entrant only bids $\underline{b}=\pi_{1}(0, k)-\pi_{0}(1, k)$ and every other bidder bids below $\underline{b}$. The license fee is $\underline{b}$ and it is easy to verify that these bids constitute an equilibrium of $G_{u}(k)$.

(ii) Let $k_{1}=0, \tilde{b} \in\left[0, \pi_{1}(n, k)\right]$ and $b=\pi_{1}(n-1, k)$. Suppose exactly $k$ entrants bid $b$, one entrant only bids $\tilde{b}$ and every other bidder bids below $\tilde{b}$. The license fee is $b_{(k+1)}=\tilde{b}$. Since $\pi_{1}(n, k)-\tilde{b} \geq 0$, no (entrant) licensee benefits from lowering his bid below $\tilde{b}$. Suppose next that a non-licensee $j$ (incumbent or entrant), bids above $b$. Then the new license fee will increase to $b=\pi_{1}(n-1, k)$ and $j$ 's industry profit is $\pi_{1}(n-1, k)$ if $j$ is an incumbent firm, and $\pi_{1}(n, k)$ if $j$ is an entrant. In both cases the industry profit does not exceed the license fee. Finally, there is no equilibrium of $G_{u}(k)$ with $k_{1}=0$ and s.t. $b_{(k+1)}>\pi_{1}(n, k)$. Otherwise, the industry profit of a licensee does not cover the license fee.

(iii) Suppose $1 \leq k_{1} \leq n-1$ and let $\tilde{b} \in\left[0, w_{i h}^{k}\left(k_{1}\right)\right]$, where by 22) $w_{i h}^{k}=\pi_{1}\left(n-k_{1}, k\right)-$ $\pi_{0}\left(n-k_{1}+1, k\right)$. Denote $b=\pi_{1}\left(n-k_{1}-1, k\right)$. Suppose exactly $k_{1}$ incumbent firms and $k_{2}$ entrants bid $b$, one entrant only bids $\tilde{b}$ and every other bidder bids below $\tilde{b}$. Then $b_{(k+1)}=\tilde{b}$ 
is the license fee. An incumbent licensee obtains

$$
\pi_{1}\left(n-k_{1}, k\right)-\tilde{b} \geq \pi_{0}\left(n-k_{1}+1, k\right) .
$$

If he lowers his bid below $\tilde{b}$ he will obtain $\pi_{0}\left(n-k_{1}+1, k\right)$. By (5) this will not benefit him. A non-licensee $j$ (incumbent or entrant) who outbids a licensee $i$ (incumbent or entrant) will increase the license fee from $\tilde{b}$ to $b=\pi_{1}\left(n-k_{1}-1, k\right)$. It is easy to verify that independently of the identity of $j$ and $i, j$ 's industry profit will not exceed $\pi_{1}\left(n-k_{1}-1, k\right)$.

Next suppose $k_{1}=n$. Let $\tilde{b} \in\left[0, \pi_{1}(0, k)-\pi_{0}(1, k)\right]$ and let $b=\pi_{1}(0, k)$. Suppose every incumbent firm and exactly $k_{2}=k-n$ entrants bid $b$. Suppose also that only one entrant bids $\tilde{b}$ and all other bidders bid below $\tilde{b}$. Then the license fee is $b_{(k+1)}=\tilde{b}$. A licensee obtains

$$
\pi_{1}(0 . k)-\tilde{b} \geq \pi_{0}(1, k) \geq 0
$$

If an incumbent licensee lowers his bid below $\tilde{b}$ he will obtain $\pi_{0}(1, k)$ and by $(6)$ he will not improve his payoff. If a non-licensee entrant $j$ outbids a licensee $i$ the new license fee will be $b=\pi_{1}(0, k)$ and again, independently of the identity of $i$, the industry profit of $j$ will not exceed $\pi_{1}(0, k)$.

Finally, for $k \geq 1$ the willingness of an incumbent firm to pay for a license is at most $w_{i h}^{k}\left(k_{1}\right)$. Thus there is no equilibrium $b^{*}$ of $G_{u}(k)$ s.t. $1 \leq k_{1} \leq n$ and $b_{(k+1)}^{*}>w_{i h}^{k}\left(k_{1}\right)$.

Proposition 2 asserts that there are multiple equilibrium points in $G_{u}(k)\left(1 \leq k<\frac{1}{\epsilon}\right)$. There are two types of multiplicity. First, any $\left(k_{1}, k_{2}\right)$ s.t. $k_{1}+k_{2}=k$ is an equilibrium outcome of $G_{u}(k)$. Second, every $\left(k_{1}, k_{2}\right)$ generates continuum of equilibrium payoffs of the innovator. In fact, if in equilibrium the highest $k+1$ bids are $\left(b_{(1)}^{*}, \ldots, b_{(k)}^{*}, b_{(k+1)}^{*}\right)$ then for every $b, 0 \leq b \leq b_{(k+1)}^{*}$, changing only the $(k+1)$ th highest bid from $b_{(k+1)}^{*}$ to $b$ will also constitute an equilibrium outcome.

The multiplicity of equilibrium outcomes is a problem even if (weakly) dominated strate- 
gies are eliminated. HJM dealt with the game $G_{u}$ with this restriction. The equilibrium analysis of $G_{u}$ is very complicated. HJM analyzed only the case where $k=1$ and some other special cases. They too found multiple equilibrium points. The conclusion is that there is no obvious way to predict the outcome of $G_{u}$ nor the choice $k$ of the innovator. To provide some comparison between the innovator's payoff in UA and in either NUA or SUA, we focus here on a specific type of equilibrium in UA, the one that for any $k$ yields the innovator the highest payoff in $G_{u}(k)$. Namely, we focus in UA on the payoff of the "luckiest" innovator. We next analyze the optimal number of licenses of the "luckiest" innovator in $G_{u}$.

Lemma 1. For any $1 \leq k \leq \frac{1}{\epsilon}$, the innovator's highest equilibrium payoff in $G_{u}(k)$ is obtained when either $k_{1}=0$ or $k_{1}=\min (k, n)$.

Proof. By Proposition 2, given an arbitrary $1 \leq k \leq \frac{1}{\epsilon}$, any $0 \leq k_{1} \leq \min (k, n)$ can emerge as an equilibrium outcome. In addition the highest payoff of the innovator is $k \pi_{1}(n, k)$ if $k_{1}=0$ and $k w_{i h}^{k}\left(k_{1}\right)$ if $1 \leq k_{1} \leq n$. It is shown in the Appendix (see A.2 that $w_{i h}^{k}\left(k_{1}\right)$ is increasing in $k_{1}$. Thus $k w_{i h}^{k}\left(k_{1}\right)$ is maximized when $k_{1}=\min (k, n)$.

Proposition 3. Suppose the innovator obtains for every $k$ the highest equilibrium payoff in $G_{u}(k)$. (i) The corresponding equilibrium number of licensees in $G_{u}$ is

$$
k_{u}^{*}(n, \epsilon)= \begin{cases}n+1 & \text { if } 0<\epsilon<g(n) \\ n & \text { if } g(n) \leq \epsilon \leq f(n) \\ \tilde{k}(n, \epsilon) & \text { if } f(n)<\epsilon<\frac{2}{n+1} \\ \frac{1}{\epsilon} & \text { if } \frac{2}{n+1} \leq \epsilon<1 .\end{cases}
$$

(ii) If $0<\epsilon<g(n)$, all licensees are entrants and if $g(n)<\epsilon<1$, all licensees are incumbent firms.

Here $\frac{1}{\epsilon} \leq \tilde{k}(n, \epsilon) \leq n$ for $f(n) \leq \epsilon \leq \frac{2}{n+1}$ and $\tilde{k}(n, \epsilon)$ is decreasing in $\epsilon$. The exact formulas of $f(n), g(n)$ and $\tilde{k}(n, \epsilon)$ are quite complicated and not revealing any insights. This is the reason they all appear in $\mathrm{A} .1$ of the Appendix. 
Remark: Let $\frac{2}{n+1} \leq \epsilon<1$ and suppose bidders do not use dominated strategies. Then the unique optimal strategy of the innovator is to auction off $k=\frac{1}{\epsilon}$ licenses. The Cournot price reduces to the pre-innovation marginal $\operatorname{cost} c$, and every non-licensee firm is driven out of the market. Consequently, the multiplicity of equilibrium points of UA occurs only when $\epsilon<\frac{2}{n+1}$.

Proof. See A.3 of the Appendix.

Proposition 3 shows that for less significant innovations the innovator obtains the highest equilibrium payoff when all licensees are entrants. Indeed an entrant licensee increases the number of active firms by 1 causing the Cournot profit of each firm to shrink. However when selling licenses only to entrants each licensee pays her entire Cournot profit for a license as opposed to the case where the innovator sells some licenses to incumbent firms (in the later case every licensee pays only the incremental profit of an incumbent licensee). When the magnitude of the innovation is relatively small the willingness to pay of an incumbent firm for a license is small and the negative effect of additional entry is offset by the incremental willingness to pay of entrants, as compared with incumbent firms. When, however, the innovation is relatively large, the benefit from having only entrant licensees cannot compensate for the loss of a stronger competition and the innovator is best off when all licensees are incumbent firms.

Corollary 1. The highest equilibrium payoff of the innovator in $G_{u}$ is

$$
\pi_{u}^{*}(n, \epsilon)= \begin{cases}(n+1) \pi_{1}(n, n+1) & \text { if } 0<\epsilon<g(n) \\ n\left(\pi_{1}(0, n)-\pi_{0}(1, n)\right) & \text { if } g(n) \leq \epsilon \leq f(n) \\ \tilde{k}\left(\pi_{1}(n-\tilde{k}, \tilde{k})-\pi_{0}(n-\tilde{k}+1, \tilde{k})\right) & \text { if } f(n)<\epsilon<\frac{2}{n+1} \\ \epsilon & \text { if } \frac{2}{n+1} \leq \epsilon<1 .\end{cases}
$$

where $\tilde{k}=\tilde{k}(n, \epsilon)$.

Proof. Follows immediately from Proposition 3 . 
We use $\pi_{u}^{*}(n, \epsilon)$ as a benchmark to compare the innovator's equilibrium payoff in UA with that obtained in NUA or SUA.

\subsection{Non-Uniform Auction}

In this section the innovator can choose and announce the number of licenses to be sold to incumbent firms $\left(0 \leq k_{1} \leq n-1\right)$ and the number of licenses to be sold to potential entrants $\left(k_{2} \geq 0\right)$. Each incumbent licensee pays the $\left(k_{1}+1\right)$ th highest bid among the incumbents' bids. Each entrant licensee pays the $\left(k_{2}+1\right)$ th highest bid among the entrants' bids. In $G_{n u}\left(k_{1}, k_{2}\right)$ the willingness to pay of each incumbent firm is $\pi_{1}\left(n-k_{1}, k_{1}+k_{2}\right)-\pi_{0}(n-$ $\left.k_{1}, k_{1}+k_{2}\right)$ and the willingness to pay of each entrant is $\pi_{1}\left(n-k_{1}, k_{1}+k_{2}\right)$. Since bidding the true valuation is a (weakly) dominant strategy for each bidder, it is assumed that bidders bid truthfully in NUA. The innovator's equilibrium payoff in $G_{n u}\left(k_{1}, k_{2}\right)$ is then uniquely determined and it is given by

$$
\pi_{n u}\left(k_{1}, k_{2}\right)=k_{1}\left(\pi_{1}\left(n-k_{1}, k_{1}+k_{2}\right)-\pi_{0}\left(n-k_{1}, k_{1}+k_{2}\right)\right)+k_{2} \pi_{1}\left(n-k_{1}, k_{1}+k_{2}\right) .
$$

The analysis of the highest incentive to innovate does not require the characterization of the equilibrium licensing strategy of the innovator in NUA.

Proposition 4. A monopoly industry maximizes the revenue of the innovator if he sells licenses by NUA.

Proposition 4 asserts that a monopoly industry provides the highest incentive to innovate if licenses are sold by NUA. The proof does not make use of the linear structure of our demand and it applies to any demand function.

Proof. Suppose there are $n, n \geq 2$ incumbent firms. Denote by $\left(k_{1}^{*}, k_{2}^{*}\right)$ the optimal licensing strategy in $G_{n u}$. Let $K_{n u}^{*}=k_{1}^{*}+k_{2}^{*}$. The innovator's highest payoff is

$$
\alpha \equiv k_{1}^{*}\left(\pi_{1}\left(n-k_{1}^{*}, K_{n u}^{*}\right)-\pi_{0}\left(n-k_{1}^{*}, K_{n u}^{*}\right)\right)+k_{2}^{*} \pi_{1}\left(n-k_{1}^{*}, K_{n u}^{*}\right) .
$$


Suppose one of the incumbent firms drops out and only $(n-1)$ incumbent firms remain.

Case 1. $k_{1}^{*} \geq 1$. Using the licensing strategy $\left(k_{1}^{*}-1, k_{2}^{*}+1\right)$, the innovator obtains

$$
\beta \equiv\left(k_{1}^{*}-1\right)\left(\pi_{1}\left(n-k_{1}^{*}, K_{n u}^{*}\right)-\pi_{0}\left(n-k_{1}^{*}, K_{n u}^{*}\right)\right)+\left(k_{2}^{*}+1\right) \pi_{1}\left(n-k_{1}^{*}, K_{n u}^{*}\right) .
$$

Clearly for $K_{n u}^{*}=\frac{1}{\epsilon}, \pi_{0}\left(n-k_{1}^{*}, K_{n u}^{*}\right)=0$ and $\alpha=\beta$. For $K_{n u}^{*}<\frac{1}{\epsilon}, \beta>\alpha$.

Case 2. Suppose $k_{1}^{*}=0$. Using the licensing strategy $\left(0, k_{2}^{*}\right)$, the innovator obtains

$$
\gamma \equiv k_{2}^{*} \pi_{1}\left(n-1, k_{2}^{*}\right) \geq k_{2}^{*} \pi_{1}\left(n, k_{2}^{*}\right) \equiv \alpha
$$

Again for $k_{2}^{*}=\frac{1}{\epsilon}, \gamma=\alpha$. For $k_{2}^{*}<\frac{1}{\epsilon}, \gamma>\alpha$

Combining Cases 1 and 2, if $K_{n u}^{*}<\frac{1}{\epsilon}$ the innovator extracts strictly higher revenue with $n-1$ than with $n$ incumbent firms. For $K_{n u}^{*}=\frac{1}{\epsilon}$, when the market size is $n-1$ the innovator obtains a payoff which is at least as high as in case where the market size is $n$. Since this is true for all $n \geq 2$, the proof is complete.

We next characterize the equilibrium of $G_{n u}$. Since $k_{1} \leq n-1$ the case $n=1$ is trivial to analyze. The equilibrium analysis of $G_{n u}$ therefore focuses only on cases $n \geq 2$.

Remark: We could extend our definition to $k_{1}=n$ if we allow the innovator to charge a fixed fee in this case. For $n=1$ this fee should be $\pi_{1}\left(0, k_{2}\right)-\pi_{0}\left(1, k_{2}\right)$. With this definition it is easy to verify that the optimal $\left(k_{1}, k_{2}\right)$ in case $n=1$ is $k_{1}=1$ and $k_{2}=0$. The analysis of NUA with this extension is tedious (see Sen and Tauman (2007)) and it will not change the basic results of this paper.

Proposition 5. The unique equilibrium licensing strategy of the innovator in $G_{n u}$ is

(i) For $n \geq 3$

$$
k_{1}^{n *}(n, \epsilon)= \begin{cases}n-1 & \text { if } 0<\epsilon \leq \frac{2}{3 n-5} \\ \frac{n+1}{4}+\frac{1}{2 \epsilon} & \text { if } \frac{2}{3 n-5} \leq \epsilon \leq \frac{2}{n+1} \\ \frac{1}{\epsilon} & \text { if } \frac{2}{n+1} \leq \epsilon<1,\end{cases}
$$




$$
k_{2}^{n *}(n, \epsilon)= \begin{cases}\frac{2(n+2 \epsilon)}{2 n \epsilon+1}-(n-1) & \text { if } 0<\epsilon \leq \frac{1}{2 n-4} \\ 0 & \text { if } \frac{1}{2 n-4} \leq \epsilon<1\end{cases}
$$

(ii) For $n=2$

$$
\begin{gathered}
k_{1}^{n *}(2, \epsilon)=1, \\
k_{2}^{n *}(2, \epsilon)= \begin{cases}\frac{3}{4 \epsilon+1} & \text { if } 0<\epsilon \leq \frac{1}{2} \\
\frac{1}{\epsilon}-1 & \text { if } \frac{1}{2} \leq \epsilon<1 .\end{cases}
\end{gathered}
$$

Proof. See A.4 of the Appendix.

Corollary 2. Let $\epsilon, 0<\epsilon<c$, be fixed and suppose that the market is competitive ( $n$ sufficiently large). Then $k_{1}^{n *}=\frac{1}{\epsilon}$ and $k_{2}^{n *}=0$. That is all licensees are incumbent firms, the market price is c and every non-licensee firm drops out of the market.

Proof. Immediate from Proposition 5 part (i).

Corollary 3. In equilibrium of $G_{n u}$ the innovator sells licenses to entrants only if he also sells licenses to all (but one) incumbent firms.

Proof. For $n \geq 3$, the claims follow from Proposition 5 part (i), the inequality $\frac{1}{2 n-4} \leq \frac{2}{3 n-5}$, and from $k_{1}^{*}(n, \epsilon)$ being decreasing in $\epsilon$. If $n=1$ or $n=2$ the claim is an immediate consequence of part (ii) of Proposition 5.

Let $K_{n u}^{*}$ be the total number of licenses the innovator sells $\left(K_{n u}^{*}=k_{1}^{n *}+k_{2}^{n *}\right)$. Corollary 3 asserts that if $0<\epsilon<\frac{1}{2 n-4}$ the innovator sells $n-1$ licenses to incumbent firms and the remaining $K_{n u}^{*}-(n-1)$ licenses he sells to entrants. If $\epsilon \geq \frac{1}{2 n-4}$ all $K_{n u}^{*}$ licensees are incumbent firms. On one hand each entrant is willing to pay all her profit for a license, but on the other hand an entrant licensee increases the number of active firms by 1 causing the Cournot profit of each firm to shrink. The effect of a weaker competition on the revenue of the innovator is larger and the innovator prefers incumbent firms on entrants. 
Corollary 4. For $n \geq 3$

$$
K_{n u}^{*}(n, \epsilon)= \begin{cases}\frac{2(n+2 \epsilon)}{2 n \epsilon+1} & \text { if } 0<\epsilon \leq \frac{1}{2 n-4} \\ n-1 & \text { if } \frac{1}{2 n-4} \leq \epsilon \leq \frac{2}{3 n-5} \\ \frac{n+1}{4}+\frac{1}{2 \epsilon} & \text { if } \frac{2}{3 n-5} \leq \epsilon \leq \frac{2}{n+1} \\ \frac{1}{\epsilon} & \text { if } \frac{2}{n+1} \leq \epsilon<1\end{cases}
$$

For $n=2$

$$
K_{n u}^{*}(n, \epsilon)= \begin{cases}\frac{3}{4 \epsilon+1}+1 & \text { if } 0<\epsilon \leq \frac{1}{2} \\ \frac{1}{\epsilon} & \text { if } \frac{1}{2} \leq \epsilon<1\end{cases}
$$

Proof. Follows immediately by Proposition 5 .

Corollary 4 asserts that the diffusion of technology is smaller for more significant innovations. In particular, for $n \geq 3$ and for relatively small $\epsilon$ the innovator sells $2 n$ licenses ( $n-1$ licenses to incumbent firms and $n+1$ licenses to new entrants). As $\epsilon$ grows the number of licenses decreases continuously to 1 , as the innovation becomes closer to a drastic innovation $(\epsilon \rightarrow 1)$. To clarify this point notice that the (negative) competition effect of additional licensee on the innovator's revenue is increasing in the magnitude of the innovation and as a result the innovator is more reluctant to issue a larger number of licenses for larger innovations. Observe that for relatively significant innovations $\left(\frac{2}{n+1} \leq \epsilon \leq 1\right.$ for $n \geq 3$ and $\frac{1}{2} \leq \epsilon<1$ for $n=2$ ) the optimal number of licenses for the innovator is the minimum number needed to drive any non-licensee firm out of the market. We next provide the innovator's equilibrium payoff in NUA.

Proposition 6. The innovator's equilibrium payoff in $G_{n u}$ is:

For $n \geq 3$

$$
\pi_{n u}^{*}(n, \epsilon)= \begin{cases}\frac{4 \epsilon^{2}+4 n \epsilon+1}{4(n+1)} & \text { if } 0<\epsilon \leq \frac{1}{2 n-4} \\ \frac{(n-1)\left(-(n-3) \epsilon^{2}+2 \epsilon\right)}{n+1} & \text { if } \frac{1}{2 n-4}<\epsilon \leq \frac{2}{3 n-5} \\ \frac{(n \epsilon+\epsilon+2)^{2}}{8(n+1)} & \text { if } \frac{2}{3 n-5} \leq \epsilon \leq \frac{2}{n+1} \\ \epsilon & \text { if } \frac{2}{n+1} \leq \epsilon<1 .\end{cases}
$$


For $n=2$

$$
\pi_{n u}^{*}(n, \epsilon)= \begin{cases}\frac{4 \epsilon^{2}+4 n \epsilon+1}{4(n+1)} & \text { if } 0<\epsilon \leq \frac{1}{2} \\ \epsilon & \text { if } \frac{1}{2} \leq \epsilon<1 .\end{cases}
$$

Corollary 1 and Proposition 6 enable us to compare the innovator's revenue in UA and NUA. In the first glance it seems that the ability to choose $\left(k_{1}, k_{2}\right)$ and to differentiate the license fee of entrants from incumbent firms should yield the innovator in NUA a higher payoff than in UA. But this may not be the case. On one hand every entrant licensee pays in NUA her entire profit (assuming zero entrant's opportunity cost) while in UA it is (like any incumbent licensee) only the incremental profit of an incumbent licensee. On the other hand an incumbent licensee is willing to pay more in UA if he takes the place of an entrant licensee and hence limit entry. In contrast, every incumbent licensee in NUA takes the place of another incumbent firm and thus does not change the number of active firms. The next proposition compares the payoff of the luckiest innovator in UA with the payoff of the innovator in NUA.

Proposition 7. Let $n \geq 3$. Then $\pi_{n u}^{*}(n, \epsilon)>\pi_{u}^{*}(n, \epsilon)$ iff $\epsilon<h(n)$.

Here $h(n) \geq g(n) \geq 0$. The formula of $h(n)$ is given in A.1 of the Appendix.

Proof. See A.6 of the Appendix.

Proposition 7 asserts that for less significant innovations, NUA yields the innovator a higher payoff compare to his highest equilibrium payoff in UA. When the magnitude of the innovation is relatively small then, as shown in propositions 3 and 5 in both types of auctions the innovator is best off having a large number of entrant licensees. In this case the benefit from collecting the entire profit of entrant licensees exceeds the loss in revenue due to lower willingness to pay of incumbent licensees. When, however, the magnitude of the innovation is relatively high, the innovator in both types of auctions is best off having small (or even 0 ) number of entrant licensees. In this case the ability to price discriminate entrant licensees contributes less to the innovator's revenue. Notice that because of the multiplicity problem 
in UA even if $\pi_{u}^{*}(n, \epsilon)>\pi_{n u}^{*}(n, \epsilon)$, there may exists other equilibrium points in UA which yields the innovator a lower payoff than in NUA. We illustrate this in the next example.

Example: Suppose $\epsilon=0.2$ and $n=5$. In NUA, the innovator's unique equilibrium payoff is $4\left(\pi_{1}(1,4)-\pi_{0}(1,4)\right)=0.213$ which is obtained when he auctions off 4 licenses only to incumbent firms. In UA the innovator's highest equilibrium payoff is $4\left(\pi_{1}(1,4)-\pi_{0}(2,4)\right)=$ 0.214, which is obtained when he auctions off 4 licenses and all winners happen also to be incumbent firms. Interestingly enough, to support this equilibrium in UA the 5th highest bid of 0.214 has to be submitted by entrants only. In this case each of the 4 incumbent licensees pays more in UA than in NUA in attempt to limit entry. However, there are other equilibrium points in UA which yields the innovator a much lower payoff. For instance there is an equilibrium in which all the 4 winners are entrants (follows by Proposition 2). In this case the innovator obtains only $4 \pi_{1}(5,4)=0.194^{4}$.

\section{$3 \quad$ Entry Vs. No Entry}

Our next goal is to compare our results with the existing literature on optimal licensing where entry is excluded. As shown in previous sections, UA has continuum of equilibrium points and there is no obvious way to predict which equilibrium will emerge. Therefore we base our study on the comparison between $G_{0}$ and $G_{n u}$, where $G_{0}$ is the game defined similarly to $G_{n u}$, but where entry is excluded.

Suppose bidders do not use dominated strategies. The willingness to pay of each bidder in $G_{0}(k), k \geq 1$, is uniquely determined and so is the innovator's equilibrium payoff. The next proposition characterizes the innovator's optimal licensing strategy in $G_{0}$.

Proposition 8. The unique equilibrium licensing strategy of the innovator in $G_{0}$ is:

\footnotetext{
${ }^{4}$ This is the highest equilibrium payoff when all 4 winners are entrants. There are other equilibrium in which the innovator ends up with zero equilibrium payoff (Proposition 2).
} 
(i) For $n \geq 3$

$$
k_{0}^{*}(n, \epsilon)= \begin{cases}n-1 & \text { if } 0<\epsilon<\frac{2}{3 n-5} \\ \frac{n+1}{4}+\frac{1}{2 \epsilon} & \text { if } \frac{2}{3 n-5} \leq \epsilon<\frac{2}{n+1} \\ \frac{1}{\epsilon} & \text { if } \frac{2}{n+1} \leq \epsilon<1 .\end{cases}
$$

(ii) For $n \leq 2$

$$
k_{0}^{*}(n, \epsilon)=n-1
$$

The proof follows from Kamien, Oren and Tauman (1992).

Observe that by Proposition 8, $k_{0}^{*}(n, \epsilon)=k_{1}^{n *}(n, \epsilon)$ where $k_{1}^{n *}(n, \epsilon)$, the optimal number of incumbent licensees in $G_{n u}$, is given in Proposition 5. This is not very surprising in light of Corollary 3. By propositions 5 and 8 for less significant innovations $\left(0<\epsilon \leq \frac{1}{2 n-4}\right)$, $k_{0}^{*}(n, \epsilon)=k_{1}^{*}(n, \epsilon)$ and $k_{2}^{*}>0$. In this case $G_{n u}$ results in a higher diffusion of technology and bigger post-innovation market size. The difference in post-innovation market size is larger for less significant magnitude of innovation.

The next proposition characterizes the innovator's revenue and the post-innovation market price in $G_{0}$.

Proposition 9. Consider the game $G_{0}$. (i) the innovator's equilibrium payoff is

For $n \geq 3$

$$
\pi_{0}^{*}(n, \epsilon)= \begin{cases}\frac{(n-1)\left(-(n-3) \epsilon^{2}+2 \epsilon\right)}{n+1} & \text { if } 0<\epsilon \leq \frac{2}{3 n-5} \\ \frac{(n \epsilon+\epsilon+2)^{2}}{8(n+1)} & \text { if } \frac{2}{3 n-5} \leq \epsilon \leq \frac{2}{n+1} \\ \epsilon & \text { if } \frac{2}{n+1} \leq \epsilon<1 .\end{cases}
$$

For $n \leq 2$

$$
\pi_{0}^{*}(n, \epsilon)=\frac{(n-1)\left(-(n-3) \epsilon^{2}+2 \epsilon\right)}{n+1}
$$

(ii) The post-innovation market price is 
For $n \geq 3$

$$
p_{0}^{*}(n, \epsilon)= \begin{cases}c+\frac{1-(n-1) \epsilon}{n+1} & \text { if } 0 \leq \epsilon \leq \frac{2}{3 n-5} \\ c+\frac{2-(n+1) \epsilon}{4(n+1)} & \text { if } \frac{2}{3 n-5} \leq \epsilon \leq \frac{2}{n+1} \\ c & \text { if } \frac{2}{n+1} \leq \epsilon<1 .\end{cases}
$$

For $n \leq 2$

$$
p_{0}^{*}(n, \epsilon)=c+\frac{1-(n-1) \epsilon}{n+1}
$$

Proof. Follows from Proposition 8

Corollary 5. Suppose $n \geq 3$. $\pi_{n u}^{*}(n, \epsilon)-\pi_{0}^{*}(n, \epsilon)$ and $p_{0}^{*}(n, \epsilon)-p_{n u}^{*}(n, \epsilon)$ are both decreasing in $n$ and decreasing in $\epsilon$.

Proof. See A.7 of the Appendix.

Corollary 5 asserts that the increment in the innovator's revenue from allowing entry is smaller when there are larger number of incumbent firms or when the magnitude of the innovation is more significant. The same result holds true for the difference in the postinnovation market price. It is shown in Corollary 6 below that these differences vanishes if either $n$ or $\epsilon$ is sufficiently large.

Corollary 6. Allowing entry will not change the innovator's revenue nor the social welfare if either (i) $\epsilon>0$ and $n$ is sufficiently large, or (ii) $n \geq 3$ and $\epsilon$ is sufficiently large.

Proof. By propositions 5 and $8 k_{0}^{*}(n, \epsilon)=k_{1}^{n *}(n, \epsilon)$ for any $n$ and $\epsilon, k_{2}^{n *}(n, \epsilon)=0$ for $n \geq 3$ and $\frac{1}{2 n-4} \leq \epsilon<1$.

Corollary 6 asserts that for any $\epsilon>0$ there is no difference in price nor in the innovator's payoff between $G_{n u}$ and $G_{0}$, for sufficiently large $n$. This is because the innovator sells licenses to entrants only if he sells licenses to all (but one) incumbent firms (Corollary 3). For a market with large number of incumbents the innovator will not sell licenses to entrants even when entry is allowed. For any $n \geq 3$, the same result holds for sufficiently large $\epsilon$. 
Next we characterize the market structure that provides the highest incentive to innovate in $G_{0}$.

Proposition 10. An oligopoly industry with size $n=\max \left(3,2 \sqrt{2+\frac{1}{\epsilon}}-1\right)$ maximizes the revenue of the innovator in $G_{0}$.

Proof. See A.8 of the Appendix.

Proposition 10 asserts that when entry is excluded, the incentive to innovate is maximized when the market is oligopoly and the optimal size is decreasing in the magnitude of the innovation (with at least 3 firms). If however the market is open to entry the incentive to innovate is maximized in a monopoly market (Proposition 4).

\section{Semi-Uniform auction}

Finally we introduce and analyze another auction mechanism, a semi-uniform auction (SUA), with a weaker asymmetry requirement than NUA. In this auction the innovator chooses $\left(k_{1}, k_{2}\right), 1 \leq k_{1} \leq n-1$ and $k_{2} \geq 0$. The $k_{1}$ highest incumbent bidders and the $k_{2}$ highest entrant bidders win the auction and all of them pay the same license fee which is the lowest winning bid5. Note that the willingness to pay of an incumbent firm is 0 if $k_{1}=n$. This is the reason we restrict our analysis to $k_{1} \leq n-1$. In SUA, like in NUA, the innovator controls the number of incumbent and the number of entrant licensees, but unlike NUA the innovator charges every licensee the fee.

Let $G_{s u}$ be the game associated with SUA. In the subgame $G_{s u}\left(k_{1}, k_{2}\right)$ of $G_{s u}$ each incumbent is willing to pay

$$
w_{l}\left(k_{1}, k_{2}\right)=\pi_{1}\left(n-k_{1}, k_{1}+k_{2}\right)-\pi_{0}\left(n-k_{1}, k_{1}+k_{2}\right) .
$$

\footnotetext{
${ }^{5}$ Note that in SUA the highest losing bid, if submitted by an entrant, may be higher than the willingness to pay of an incumbent winner. To avoid this problem we define the license fee as the lowest winning bid.
} 
Each entrant is willing to pay

$$
w_{e}\left(k_{1}, k_{2}\right)=\pi_{1}\left(n-k_{1}, k_{1}+k_{2}\right) .
$$

Clearly $w_{l}\left(k_{1}, k_{2}\right) \leq w_{e}\left(k_{1}, k_{2}\right)$. In particular, for $k_{1}+k_{2}<\frac{1}{\epsilon}, \pi_{0}\left(n-k_{1}, k_{1}+k_{2}\right)>0$ and $w_{l}\left(k_{1}, k_{2}\right)<w_{e}\left(k_{1}, k_{2}\right)$. It can be verified that the innovator's equilibrium payoff in $G_{s u}\left(k_{1}, k_{2}\right)$ is uniquely determined and it is $\left(k_{1}+k_{2}\right) w_{l}\left(k_{1}, k_{2}\right)$ for $k_{1}>0$ and $k_{2} w_{e}\left(0, k_{2}\right)$ for $k_{1}=0$.

Remark: Notice that some entrants may bid above the SUA license fee and still do not obtain a license. Yet in equilibrium the innovator has no incentive to increase $k_{2}$ since it will increase competition and lower his total revenue.

Let $\pi_{s u}^{*}(n, \epsilon)$ be the innovator's equilibrium payoff in $G_{s u}$.

$$
\pi_{s u}^{*}(n, \epsilon)=\max \left(\pi_{s u}^{0}(n, \epsilon), \hat{\pi}_{s u}(n, \epsilon)\right)
$$

where

$$
\pi_{s u}^{0}(n, \epsilon)=\max _{k_{2} \geq 1} k_{2} w_{e}\left(0, k_{2}\right)
$$

and

$$
\hat{\pi}_{s u}(n, \epsilon)=\max _{\substack{1 \leq k_{1} \leq n-1 \\ 0 \leq k_{2}}}\left(k_{1}+k_{2}\right) w_{l}\left(k_{1}, k_{2}\right) .
$$

When $k_{1}=0$ each entrant licensee pays her entire profit for a license. But when $k_{1}>0$ each entrant licensee pays less, only the willingness to pay of an incumbent licensee.

Proposition 11. (i) $\pi_{s u}^{*}(n, \epsilon) \leq \pi_{u}^{*}(n, \epsilon)$ and (ii) $\pi_{s u}^{*}(n, \epsilon) \leq \pi_{n u}^{*}(n, \epsilon)$.

Proof. (i) In UA the highest equilibrium payoff of the innovator is

$$
\pi_{u}^{*}(n, \epsilon)=\max \left(\pi_{u}^{0}(n, \epsilon), \hat{\pi}_{u}(n, \epsilon)\right)
$$

where $\pi_{u}^{0}(n, \epsilon)=\max _{k \geq 1} k w_{e}(0, k)$ and $\hat{\pi}_{u}(n, \epsilon)=\max _{\substack{\leq k_{1} \leq n-1 \\ 0 \leq k_{2}}}\left(k_{1}+k_{2}\right) w_{h}\left(k_{1}, k_{2}\right)$. Part (i) 
follows from $w_{h}\left(k_{1}, k_{2}\right) \geq w_{l}\left(k_{1}, k_{2}\right)$ for any $\left(k_{1}, k_{2}\right)$.

(ii) Follows from the fact that for any $\left(k_{1}, k_{2}\right)$, NUA yields the innovator a higher payoff than SUA.

The innovator in UA can choose only $k$ while in SUA he can choose in addition the partition of $k$. In the first glance the innovator should always obtain a higher payoff in SUA than in UA. But this is not necessarily the case. There are cases in which UA yields the innovator a higher payoff than SUA since an incumbent licensee is willing to pay more in UA for further entry prevention. As for the comparison between SUA and NUA, note that in NUA, in addition to choosing the partition $\left(k_{1}, k_{2}\right)$, the innovator can discriminate in price entrants from incumbent licensees. Therefore for any $\left(k_{1}, k_{2}\right)$ and $k_{2}>0$, NUA yields the innovator a higher payoff than SUA.

Next we characterize the market structure that provides the highest incentive to innovate in $G_{s u}$. Like in NUA, in SUA the innovator obtains the highest payoff in a monopoly market.

Proposition 12. A monopoly industry maximizes the revenue of the innovator if he sells licenses by SUA.

Proof. The proof is similar to that of Proposition 4, and hence omitted.

We next analyze for any industry size $n$ the optimal licensing strategy of the innovator in SUA as a function of $\epsilon$. Unlike NUA, the equilibrium licensing strategy is discontinuous for one value of $\epsilon$ (the equilibrium revenue of the innovator is however continuous for any $\epsilon$ ). We will discuss this point after stating the next proposition.

Proposition 13. Consider the equilibrium of $G_{s u}$. For $n \geq 3$ there exists $r(n), r(n)>0$, such that (i) if $r(n)<\epsilon \leq 1$ then the innovator sells positive number of licenses to entrants only if he sells $n-1$ licenses to all (but 1) incumbent firms. In this region the total number of licensees is larger, the less significant is the magnitude of innovation. (ii) At $\epsilon=r(n)$ the innovator has two optimal licensing strategies: either selling $n-1$ licenses to incumbent firms 
and some licenses to entrants, or selling $n+1$ licenses to only entrants. (iii) if $0<\epsilon<r(n)$, the innovator sells $n+1$ licenses to entrants only.

The proof as well as the exact formula of the equilibrium strategy of the innovator in SUA appears in A.10 of the Appendix.

The reason for selling licenses only to entrant in SUA for less significant innovations is the ability of the innovator to extract the entire industry profit of every entrant licensee. This is in contrast to the case where he sells some licenses also to incumbent firms. In the latter case the license fee an entrant pays is equal to the willingness to pay of an incumbent licensee which decreases to zero as $\epsilon \rightarrow 0$. To illustrate this point suppose that $\epsilon=q^{6}$. In this case if the innovator sells some licenses to incumbent firms, every licensee in SUA will pay zero license fee to the innovator. If instead, the innovator sells licenses only to entrants, he obtains the entire industry profit of all new entrant licensees (entrants wouldn't be able to enter the market otherwise). If there are $n$ incumbent firms the linear demand assumption implies that the innovator maximizes his revenue if the number of entrant licensees is $n+1$.

Let us compare the outcome of SUA with the outcome of NUA. First observe that for $\epsilon>r(n)$ in both SUA and NUA the total number of licenses the innovator sells is decreasing in the magnitude of the innovation and the innovator may sell licenses to entrants, only if he also sells licenses to all (but 1) incumbent firms. The main difference between SUA and NUA is when $\epsilon<r(n)$. In this case, unlike NUA, the innovator in SUA sells licenses only to new entrants and not to incumbent firms. This shift in the innovator's optimal strategy generates a discontinuity in the number of licenses at $\epsilon=r(n)$. In contrast, the innovator in NUA can discriminate the entrant licensees and can extract their entire industry profit whether or not he sells licenses to incumbent firms. Therefore in NUA the innovator sells licenses to both new entrants and incumbent firms, even for small $\epsilon$.

Let $K_{s u}^{*}$ and $K_{n u}^{*}$ be the total number of licenses the innovator sells in SUA and NUA, respectively.

\footnotetext{
${ }^{6}$ This is the case where the innovator provides no improvement in cost but his technology allows free entry.
} 
Proposition 14. Suppose $n \geq 2$. There exists $l(n), 0<l(n)<1$, such that if $0<\epsilon \leq l(n)$, $K_{n u}^{*}(n, \epsilon)>K_{s u}^{*}(n, \epsilon)$.

Proof. See A.13 of the Appendix.

Proposition 14 shows that comparing with SUA, NUA results in higher diffusion of technology for less significant innovations. As shown in Proposition 13 for less significant innovations, the innovator in SUA does not sell licenses to incumbent firms while in NUA he sells licenses to entrants in addition to all (but 1) incumbent firms. Therefore the ability to price discriminate new entrant licensees has positive effect not only on the innovator's revenue but also on social welfare, as compare to $\mathrm{SUA}^{7}$.

\section{References}

[1] Kenneth Arrow. "Economic welfare and the allocation of resources for invention". In: The rate and direction of inventive activity: Economic and social factors. Princeton University Press, 1962, pp. 609-626.

[2] Yongmin Chen and Marius Schwartz. "Product innovation incentives: monopoly vs. competition". In: Journal of Economics $\&$ Management Strategy 22.3 (2013), pp. 513528.

[3] Heidrun C Hoppe, Philippe Jehiel, and Benny Moldovanu. "License auctions and market structure". In: Journal of Economics $\&$ Management Strategy 15.2 (2006), pp. 371396.

[4] Morton I Kamien. "Patent licensing". In: Handbook of game theory with economic applications 1 (1992), pp. 331-354.

\footnotetext{
${ }^{7}$ When $\epsilon \leq l(n)$ the market price in SUA is $c+\frac{1-(n+1) \epsilon}{2(n+1)}$ (easy to verify) while the market price in NUA is $c+\frac{1-2 \epsilon}{2(n+1)}$ (Proposition 6).
} 
[5] Morton I Kamien, Shmuel S Oren, and Yair Tauman. "Optimal licensing of costreducing innovation". In: Journal of Mathematical Economics 21.5 (1992), pp. 483508.

[6] Morton I Kamien and Yair Tauman. "Fees versus royalties and the private value of a patent". In: The Quarterly Journal of Economics (1986), pp. 471-491.

[7] Morton I Kamien and Yair Tauman. The private value of a patent: a game theoretic analysis. Springer, 1984.

[8] Michael L Katz. "An analysis of cooperative research and development". In: The Rand Journal of Economics (1986), pp. 527-543.

[9] Michael L Katz and Carl Shapiro. "On the licensing of innovations". In: The RAND Journal of Economics (1985), pp. 504-520.

[10] Joseph Alois Schumpeter. Socialism, capitalism and democracy. Harper and Brothers, 1942.

[11] Debapriya Sen and Yair Tauman. "General licensing schemes for a cost-reducing innovation". In: Games and Economic Behavior 59.1 (2007), pp. 163-186.

[12] Yair Tauman, Yoram Weiss, and Chang Zhao. "Bargaining in Patent Licensing with Inefficient Outcomes". Discussion Paper (2016). 


\section{A Appendix}

A.1

$$
\begin{gathered}
f(n)=\frac{n^{3}+n^{2}+2 n+4+\sqrt{n^{6}+8 n^{5}+30 n^{4}+56 n^{3}+50 n^{2}+20 n+4}}{3 n^{4}+8 n^{3}+10 n^{2}+4 n-4} \\
\tilde{k}(n, \epsilon)=\frac{2 n^{3} \epsilon+10 n^{2} \epsilon+16 n \epsilon+4 n+8 \epsilon+6-\sqrt{4 n^{6} \epsilon^{2}+34 n^{5} \epsilon^{2}+119 n^{4} \epsilon^{2}+4 n^{4} \epsilon+220 n^{3} \epsilon^{2}+26 n^{3} \epsilon+227 n^{2} \epsilon^{2}+62 n^{2} \epsilon+124 n \epsilon^{2}+4 n^{2}+64 n \epsilon+28 \epsilon^{2}+12 n+24 \epsilon+9}}{3(2 n+3) \epsilon} \\
g(n)=\max \left(0, \frac{3 n^{4}+6 n^{3}+7 n^{2}+4 n-4-2 \sqrt{n^{8}+9 n^{7}+31 n^{6}+49 n^{5}+29 n^{4}-9 n^{3}-16 n^{2}-4 n}}{5 n^{5}+15 n^{4}+19 n^{3}+9 n^{2}+4}\right) \\
h(n)=\max \left(0, \frac{n^{4}+n^{3}+2 n^{2}+4 n-\sqrt{3 n^{7}+14 n^{6}+18 n^{5}+7 n^{4}+24 n^{3}+40 n^{2}-16}}{n^{5}+2 n^{4}+n^{3}+n^{2}+4 n+4}\right) \\
r(n)=\left\{\begin{array}{c}
\text { if } n \geq 17 \\
e_{1}(n) \quad \begin{array}{l}
\text { if } 2 \leq n \leq 16 \\
f_{1}^{-1}(n)
\end{array}
\end{array}\right.
\end{gathered}
$$

where

$$
e_{1}(n)=\frac{3 n-5-2 \sqrt{n^{2}-4 n+3}}{5 n^{2}-14 n+13}
$$

and

$$
f_{1}(\epsilon)=-\frac{8 \sqrt{1+2} \epsilon \epsilon^{3 / 2}-11 \epsilon^{2}+2 \sqrt{-48 \sqrt{1+2} \epsilon \epsilon^{7 / 2}-12 \sqrt{1+2 \epsilon} \epsilon^{5 / 2}+68 \epsilon^{4}+34 \epsilon^{3}+2 \epsilon^{2}}-3 \epsilon}{\epsilon^{2}}
$$

Figure 1 shows that the inverse function of $f_{1}(\epsilon)$ exists for $0<\epsilon<\frac{1}{2}$. 


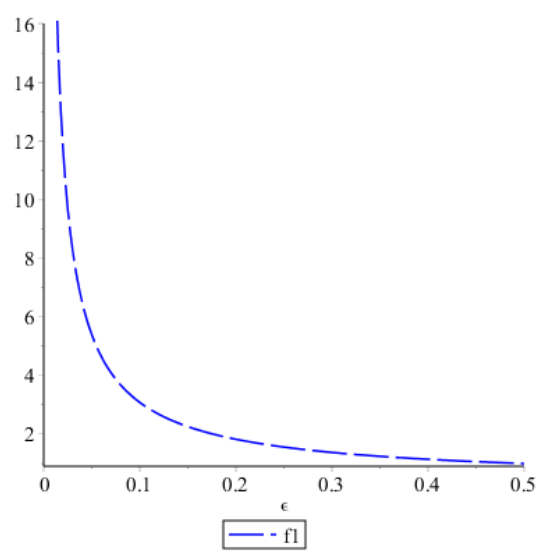

Figure 1: The value of $f_{1}(\epsilon)$

\section{A.2 Proof of $\pi_{1}\left(n-k_{1}, k\right)-\pi_{0}\left(n-k_{1}+1, k\right)$ being increasing in $k_{1}$}

Let $m=n-k_{1}$, we will show that $\pi_{1}(m, k)-\pi_{0}(m+1, k)$ is decreasing in $m$.

$$
\pi_{1}(m, k)-\pi_{0}(m+1, k)=\frac{(1+(m+1) \epsilon)^{2}}{(m+k+1)^{2}}-\frac{(1-k \epsilon)^{2}}{(m+k+2)^{2}}
$$

The first order condition (using Maple) is

$$
\frac{\partial\left(\pi_{1}(m, k)-\pi_{0}(m+1, k)\right)}{\partial m}=G \epsilon^{2}+H \epsilon+I
$$

where

$G=\frac{2 k^{5}+8 k^{4} m+12 k^{3} m^{2}+8 k^{2} m^{3}+2 k m^{4}+8 k^{4}+30 k^{3} m+36 k^{2} m^{2}+14 k m^{3}+18 k^{3}+54 k^{2} m+36 k m^{2}+26 k^{2}+40 m k+16 k}{(m+k+1)^{3}(m+2+k)^{3}}>0$

$H=\frac{-2 k^{4}-8 k^{3} m-12 k^{2} m^{2}-8 k m^{3}-2 m^{4}-2 k^{3}-18 k^{2} m-30 k m^{2}-14 m^{3}-36 m k-36 m^{2}-12 k-40 m-16}{(m+k+1)^{3}(m+2+k)^{3}}$

and

$$
I=\frac{-6 k^{2}-12 m k-6 m^{2}-18 k-18 m-14}{(m+k+1)^{3}(m+2+k)^{3}}
$$

Therefore $\frac{\partial\left(\pi_{1}(m, k)-\pi_{0}(m+1, k)\right)}{\partial m}$ is in quadratic in $\epsilon$ with $G>0$. 
The equation $\frac{\partial\left(\pi_{1}(m, k)-\pi_{0}(m+1, k)\right)}{\partial m}=0$ has two solutions in $\epsilon$.

$$
\epsilon_{1}=-\frac{3 k^{2}+6 m k+3 m^{2}+9 k+9 m+7}{k^{4}+4 k^{3} m+6 k^{2} m^{2}+4 k m^{3}+m^{4}+4 k^{3}+15 k^{2} m+18 k m^{2}+7 m^{3}+9 k^{2}+27 m k+18 m^{2}+13 k+20 m+8}<0
$$

and

$$
\epsilon_{2}=\frac{1}{k}>0
$$

Therefore for $0<\epsilon \leq \frac{1}{k}, \frac{\partial\left(\pi_{1}(m, k)-\pi_{0}(m+1, k)\right)}{\partial m}<0$ and $\pi_{1}(m, k)-\pi_{0}(m+1, k)$ is decreasing in $m$. Since $m=n-k_{1}, \pi_{1}\left(n-k_{1}, k\right)-\pi_{0}\left(n-k_{1}+1, k\right)$ is increasing in $k_{1}$.

\section{A.3 Proof of Proposition 3}

By Lemma 1 the highest equilibrium payoff of the innovator in $G_{u}$ is

$$
\pi_{u}^{*}(n, \epsilon)=\max \left(\pi^{0}(n, \epsilon), \hat{\pi}(n, \epsilon)\right)
$$

where $\pi^{0}(n, \epsilon)=\max _{k \geq 1} k \pi_{1}(n, k)$ and $\hat{\pi}(n, \epsilon)=\max _{k \geq 1} k\left(\pi_{1}(n-\min (k, n), k)-\pi_{0}(n-\right.$ $\min (k, n)+1, k))$.

Let $k_{u}^{*}(n, \epsilon), k^{0}(n, \epsilon)$ and $\hat{k}(n, \epsilon)$ be maximizers of $\pi_{u}^{*}(n, \epsilon), \pi^{0}(n, \epsilon)$ and $\hat{\pi}(n, \epsilon)$, respectively. Clearly either $k^{0}(n, \epsilon)$ or $\hat{k}(n, \epsilon)$ is a maximizer of $\pi_{u}^{*}(n, \epsilon)$.

Lemma 2. $k\left(\pi_{1}(0, k)-\pi_{0}(1, k)\right)$ is decreasing in $k$.

Proof. Let

$$
J=k\left(\pi_{1}(0, k)-\pi_{0}(1, k)\right)=k\left(\left(\frac{1+\epsilon}{1+k}\right)^{2}-\left(\frac{1-k \epsilon}{2+k}\right)^{2}\right)
$$

Then

$$
\frac{\partial J}{\partial k}=A \epsilon^{2}+B \epsilon+C
$$


where $A, B$ and $C$ are functions of $k$. In particular,

$$
\begin{gathered}
A=-\frac{k^{6}+9 k^{5}+22 k^{4}+24 k^{3}+12 k^{2}-4 k-8}{(1+k)^{3}(2+k)^{3}} \\
B=-\frac{-6 k^{4}-14 k^{3}-12 k^{2}-16 k-16}{(1+k)^{3}(2+k)^{3}} \\
C=-\frac{4 k^{3}+9 k^{2}+k-6}{(1+k)^{3}(2+k)^{3}}
\end{gathered}
$$

and

$$
B^{2}-4 A C=\frac{-16\left(k^{3}+k^{2}-2 k-1\right)}{(k+2)^{4}(1+k)^{2}}
$$

Clearly $A<0$ for $k \geq 1$ and $B^{2}-4 A C<0$ for $k \geq 2$. Therefore $\frac{\partial J}{\partial k}<0$ for $k \geq 2$ and $J$ is maximized either at $k=1$ or at $k=2$.

Since $\left.J\right|_{k=1}=\frac{1}{36}(\epsilon+5)(5 \epsilon+1)$ and $\left.J\right|_{k=2}=-\frac{1}{72}(10 \epsilon+1)(2 \epsilon-7)$, for every $\epsilon$

$$
\left.J\right|_{k=1}-\left.J\right|_{k=2}=\frac{5}{12} \epsilon^{2}-\frac{2}{9} \epsilon+\frac{1}{24}>0
$$

Thus $J=k\left(\pi_{1}(0, k)-\pi_{0}(1, k)\right)$ is decreasing in $k$ for $k \geq 1$.

Lemma 3. (i) $k^{0}(n, \epsilon) \leq n+1$ and (ii) $\hat{k}(n, \epsilon) \leq n$.

Proof. (i) By (1) and Proposition 1

$$
\pi^{0}(n, \epsilon)=\max _{1 \leq k \leq \frac{1}{\epsilon}} \frac{k(1+(n+1) \epsilon)^{2}}{(n+k+1)^{2}}
$$

and it is maximized at $k=\min \left(n+1, \frac{1}{\epsilon}\right)$. Hence $k^{0}(n, \epsilon) \leq n+1$.

(ii) Let

$$
\hat{\pi}_{1}(n, \epsilon)=\max _{1 \leq k<n} k\left(\pi_{1}(n-k, k)-\pi_{0}(n-k+1, k)\right)
$$

and

$$
\hat{\pi}_{2}(n, \epsilon)=\max _{k \geq n} k\left(\pi_{1}(0, k)-\pi_{0}(1, k)\right)
$$


Then $\hat{\pi}(n, \epsilon)=\max \left(\hat{\pi}_{1}(n, \epsilon), \hat{\pi}_{2}(n, \epsilon)\right)$. But $k\left(\pi_{1}(0, k)-\pi_{0}(1, k)\right)$ is decreasing in $k$ (Lemma 2. This implies $\hat{k}(n, \epsilon) \leq n$.

The next lemma characterizes both $k^{0}(n, \epsilon)$ and $\hat{k}(n, \epsilon)$.

\section{Lemma 4.}

$$
k^{0}(n, \epsilon)= \begin{cases}n+1 & \text { if } 0<\epsilon<\frac{1}{n+1} \\ \frac{1}{\epsilon} & \text { if } \frac{1}{n+1} \leq \epsilon<1\end{cases}
$$

and

$$
\hat{k}(n, \epsilon)= \begin{cases}n & \text { if } 0<\epsilon<f(n) \\ \tilde{k}(n, \epsilon) & \text { if } f(n) \leq \epsilon \leq \frac{2}{n+1} \\ \frac{1}{\epsilon} & \text { if } \frac{2}{n+1}<\epsilon<1\end{cases}
$$

where

$$
f(n)=\frac{n^{3}+n^{2}+2 n+4+\sqrt{n^{6}+8 n^{5}+30 n^{4}+56 n^{3}+50 n^{2}+20 n+4}}{3 n^{4}+8 n^{3}+10 n^{2}+4 n-4},
$$

$\tilde{k}(n, \epsilon)=\frac{2 n^{3} \epsilon+10 n^{2} \epsilon+16 n \epsilon+4 n+8 \epsilon+6-\sqrt{4 n^{6} \epsilon^{2}+34 n^{5} \epsilon^{2}+119 n^{4} \epsilon^{2}+4 n^{4} \epsilon+220 n^{3} \epsilon^{2}+26 n^{3} \epsilon+227 n^{2} \epsilon^{2}+62 n^{2} \epsilon+124 n \epsilon^{2}+4 n^{2}+64 n \epsilon+28 \epsilon^{2}+12 n+24 \epsilon+9}}{3(2 n+3) \epsilon}$.

Here $\frac{1}{\epsilon} \leq \tilde{k}(n, \epsilon) \leq n$ for $f(n) \leq \epsilon \leq \frac{2}{n+1}$ and $\tilde{k}(n, \epsilon)$ is decreasing in $\epsilon$.

Proof. 14 follows from the proof of part (i) of Lemma 3 . We next analyze $\hat{k}(n, \epsilon)$.

By part (ii) of Lemma $3, \hat{k}(n, \epsilon) \leq n$. Then

$$
\begin{aligned}
\hat{\pi}(n, \epsilon) & =\max _{1 \leq k \leq n} k\left(\pi_{1}(n-k, k)-\pi_{0}(n-k+1, k)\right) \\
& =k\left(\frac{(1+(n-k+1) \epsilon)^{2}}{(n+1)^{2}}-\frac{(1-k \epsilon)^{2}}{(n+2)^{2}}\right)
\end{aligned}
$$

The first order condition is

$$
\frac{\partial \hat{\pi}(n, \epsilon)}{\partial k}=D k^{2}+E k+F
$$

where

$$
D=\frac{6 n \epsilon^{2}+9 \epsilon^{2}}{(n+1)^{2}(n+2)^{2}}>0
$$




$$
\begin{gathered}
E=\frac{-4 n^{3} \epsilon^{2}-20 n^{2} \epsilon^{2}-32 n \epsilon^{2}-8 n \epsilon-16 \epsilon^{2}-12 \epsilon}{(n+1)^{2}(n+2)^{2}} \\
F=\frac{n^{4} \epsilon^{2}+6 n^{3} \epsilon^{2}+2 n^{3} \epsilon+13 n^{2} \epsilon^{2}+10 n^{2} \epsilon+12 n \epsilon^{2}+16 n \epsilon+4 \epsilon^{2}+2 n+8 \epsilon+3}{(n+1)^{2}(n+2)^{2}}
\end{gathered}
$$

and

$E^{2}-4 D F=4 \frac{\epsilon^{2}\left(4 n^{6} \epsilon^{2}+34 n^{5} \epsilon^{2}+119 n^{4} \epsilon^{2}+4 n^{4} \epsilon+220 n^{3} \epsilon^{2}+26 n^{3} \epsilon+227 n^{2} \epsilon^{2}+62 n^{2} \epsilon+124 n \epsilon^{2}+4 n^{2}+64 n \epsilon+28 \epsilon^{2}+12 n+24 \epsilon+9\right)}{(n+1)^{4}(n+2)^{4}}>0$

Let $c_{1}$ and $c_{2}$ be the solution in $k$ of the quadratic function $\frac{\partial \hat{\pi}(n, \epsilon)}{\partial k}=0$. Then

$$
\begin{aligned}
& c_{1}=\frac{2 n^{3} \epsilon+10 n^{2} \epsilon+16 n \epsilon+4 n+8 \epsilon+6-\sqrt{4 n^{6} \epsilon^{2}+34 n^{5} \epsilon^{2}+119 n^{4} \epsilon^{2}+4 n^{4} \epsilon+220 n^{3} \epsilon^{2}+26 n^{3} \epsilon+227 n^{2} \epsilon^{2}+62 n^{2} \epsilon+124 n \epsilon^{2}+4 n^{2}+64 n \epsilon+28 \epsilon^{2}+12 n+24 \epsilon+9}}{3(2 n+3) \epsilon} \\
& c_{2}=\frac{2 n^{3} \epsilon+10 n^{2} \epsilon+16 n \epsilon+4 n+8 \epsilon+6+\sqrt{4 n^{6} \epsilon^{2}+34 n^{5} \epsilon^{2}+119 n^{4} \epsilon^{2}+4 n^{4} \epsilon+220 n^{3} \epsilon^{2}+26 n^{3} \epsilon+227 n^{2} \epsilon^{2}+62 n^{2} \epsilon+124 n \epsilon^{2}+4 n^{2}+64 n \epsilon+28 \epsilon^{2}+12 n+24 \epsilon+9}}{3(2 n+3) \epsilon}
\end{aligned}
$$

It can be easily verified that when $\epsilon \geq 0$ and $n \geq 1, c_{1}>0$. Next we compare $c_{1}$ with $\frac{1}{\epsilon}$.

$$
\frac{1}{\epsilon}-c_{1}=\frac{s(n, \epsilon)-t(n, \epsilon)}{3(2 n+3) \epsilon}
$$

where

$$
s(n, \epsilon)=\sqrt{4 n^{6} \epsilon^{2}+34 n^{5} \epsilon^{2}+119 n^{4} \epsilon^{2}+4 n^{4} \epsilon+220 n^{3} \epsilon^{2}+26 n^{3} \epsilon+227 n^{2} \epsilon^{2}+62 n^{2} \epsilon+124 n \epsilon^{2}+4 n^{2}+64 n \epsilon+28 \epsilon^{2}+12 n+24 \epsilon+9}
$$

and

$$
t(n, \epsilon)=2 n^{3} \epsilon+10 n^{2} \epsilon+16 n \epsilon+8 \epsilon-2 n-3
$$

For $\epsilon \geq 0, s(n, \epsilon)>0$ and it can be easily verified that $t(n, \epsilon) \leq 0$ iff $\epsilon \leq \frac{2 n+3}{2(n+1)(n+2)^{2}}$. By (17) for $\epsilon \leq \frac{2 n+3}{2(n+1)(n+2)^{2}}, c_{1} \leq \frac{1}{\epsilon}$. If, however, $\epsilon>\frac{2 n+3}{2(n+1)(n+2)^{2}}$ by $18 t(n, \epsilon)>0$. It can be easily verified that

$$
(s(n, \epsilon))^{2} \geq(t(n, \epsilon))^{2} \quad \text { iff } \quad 0 \leq \epsilon \leq \frac{2}{n+1}
$$

and for all $n \geq 1$, in which case, again, $c_{1} \leq \frac{1}{\epsilon}$. It can also be verified that $\frac{2}{n+1}>\frac{2 n+3}{2(n+1)(n+2)^{2}}$ 
for $n \geq 1$. Therefore $c_{1}>\frac{1}{\epsilon}$ iff $\epsilon>\frac{2}{n+1}$. Since the optimal $k$ is bounded above by $\frac{1}{\epsilon}$ (Proposition 11, for $\epsilon>\frac{2}{n+1}, \hat{k}(n, \epsilon)=\frac{1}{\epsilon}$. Next we analyze the case $0 \leq \epsilon \leq \frac{2}{n+1}$ (or equivalently $c_{1} \leq \frac{1}{\epsilon}$ ). We first compare the value of $\frac{1}{\epsilon}$ and $c_{2}$.

$$
\frac{1}{\epsilon}-c_{2}=\frac{-s(n, \epsilon)-t(n, \epsilon)}{3(2 n+3) \epsilon}
$$

as shown above, $t(n, \epsilon) \geq 0$ iff $\epsilon \geq \frac{2 n+3}{2(n+1)(n+2)^{2}}$. For $\frac{2 n+3}{2(n+1)(n+2)^{2}} \leq \epsilon \leq \frac{2}{n+1}, c_{2} \geq \frac{1}{\epsilon}$. Since $(s(n, \epsilon))^{2} \geq(t(n, \epsilon))^{2}$ for $0 \leq \epsilon<\frac{2 n+3}{2(n+1)(n+2)^{2}}$, again $c_{2} \geq \frac{1}{\epsilon}$. Thus for any $\epsilon \leq \frac{2}{n+1}, c_{2} \geq \frac{1}{\epsilon}$.

This together with (16) imply that $\hat{\pi}(n, \epsilon)$ is maximized at $k=c_{1}$.

Finally we compare the value of $c_{1}$ with $n$.

$$
n-c_{1}=\frac{s(n, \epsilon)-\left(2 n^{3} \epsilon+4 n^{2} \epsilon+7 \epsilon n+4 n+8 \epsilon+6\right)}{3(2 n+3) \epsilon}
$$

It can be easily verified that

$$
\begin{aligned}
& (s(n, \epsilon))^{2}-\left(2 n^{3} \epsilon+4 n^{2} \epsilon+7 \epsilon n+4 n+8 \epsilon+6\right)^{2}= \\
& \left(18 n^{5}+75 n^{4}+132 n^{3}+114 n^{2}+12 n-36\right) \epsilon^{2}-\left(12 n^{4}+30 n^{3}+42 n^{2}+84 n+72\right) \epsilon-\left(12 n^{2}+36 n+27\right)
\end{aligned}
$$

Thus $c_{1} \geq n$ iff the last term $\leq 0$. The solution of this quadratic inequality is

$$
\frac{n^{3}+n^{2}+2 n+4-\sqrt{n^{6}+8 n^{5}+30 n^{4}+56 n^{3}+50 n^{2}+20 n+4}}{3 n^{4}+8 n^{3}+10 n^{2}+4 n-4} \leq \epsilon \leq \frac{n^{3}+n^{2}+2 n+4+\sqrt{n^{6}+8 n^{5}+30 n^{4}+56 n^{3}+50 n^{2}+20 n+4}}{3 n^{4}+8 n^{3}+10 n^{2}+4 n-4} \equiv f(n)
$$

It can be easily verified that $\frac{n^{3}+n^{2}+2 n+4-\sqrt{n^{6}+8 n^{5}+30 n^{4}+56 n^{3}+50 n^{2}+20 n+4}}{3 n^{4}+8 n^{3}+10 n^{2}+4 n-4}<0$ for $n \geq 1$. It can also be verified that $f(n) \leq \frac{2}{n+1}$ for $n \geq 1$.

Consequently, for $0 \leq \epsilon \leq f(n), n \leq c_{1} \leq \frac{1}{\epsilon}$ and $\hat{k}(n, \epsilon)=n$; for $f(n)<\epsilon \leq \frac{2}{n+1}, c_{1}<n$, $c_{1}<\frac{1}{\epsilon}$ and $\hat{k}(n, \epsilon)=c_{1}$; for $\frac{2}{n+1} \leq \epsilon \leq 1, \frac{1}{\epsilon}<c_{1}<n$ and $\hat{k}(n, \epsilon)=\frac{1}{\epsilon}$. It is left to show that $c_{1}$ is decreasing in $\epsilon$. We first compute the first order derivative of $c_{1}$ with respect to $\epsilon$.

$$
\frac{\partial c_{1}}{\partial \epsilon}=\frac{n^{3} \epsilon+5 n^{2} \epsilon+8 \epsilon n-2 \sqrt{4 n^{6} \epsilon^{2}+34 n^{5} \epsilon^{2}+119 n^{4} \epsilon^{2}+4 n^{4} \epsilon+220 n^{3} \epsilon^{2}+26 n^{3} \epsilon+227 n^{2} \epsilon^{2}+62 n^{2} \epsilon+124 n \epsilon^{2}+4 n^{2}+64 \epsilon n+28 \epsilon^{2}+12 n+24 \epsilon+9}+2 n+4 \epsilon+3}{3 \epsilon^{2} \sqrt{4 n^{6} \epsilon^{2}+34 n^{5} \epsilon^{2}+119 n^{4} \epsilon^{2}+4 n^{4} \epsilon+220 n^{3} \epsilon^{2}+26 n^{3} \epsilon+227 n^{2} \epsilon^{2}+62 n^{2} \epsilon+124 n \epsilon^{2}+4 n^{2}+64 \epsilon n+28 \epsilon^{2}+12 n+24 \epsilon+9}}
$$


It can be easily verified that $\frac{\partial c_{1}}{\partial \epsilon}<0$ for $n \geq 1$ and $\epsilon>0$. The proof of Lemma 4 is complete.

We are now ready to characterize the equilibrium number of licensees in $G_{u}$, for the "lucky" innovator.

Case 1: Suppose $0 \leq \epsilon \leq \min \left(\frac{1}{n+1}, f(n)\right)$, then $k^{0}(n, \epsilon)=n+1$ and $\hat{k}(n, \epsilon)=n$.

$$
\begin{aligned}
\pi^{0}(n, \epsilon)-\hat{\pi}(n, \epsilon) & =(n+1) \pi_{1}(n, n+1)-n\left(\pi_{1}(0, k)-\pi_{0}(1, k)\right) \\
& =\frac{\left(5 n^{5}+15 n^{4}+19 n^{3}+9 n^{2}+4\right) \epsilon^{2}-\left(6 n^{4}+12 n^{3}+14 n^{2}+8 n-8\right) \epsilon+n^{3}-3 n^{2}-4 n+4}{4(n+1)^{2}(n+2)^{2}}
\end{aligned}
$$

It is easy to verify that $\pi^{0}(n, \epsilon) \leq \hat{\pi}(n, \epsilon)$ iff

$$
\frac{3 n^{4}+6 n^{3}+7 n^{2}+4 n-4-2 \sqrt{n^{8}+9 n^{7}+31 n^{6}+49 n^{5}+29 n^{4}-9 n^{3}-16 n^{2}-4 n}}{5 n^{5}+15 n^{4}+19 n^{3}+9 n^{2}+4} \leq \epsilon \leq \frac{3 n^{4}+6 n^{3}+7 n^{2}+4 n-4+2 \sqrt{n^{8}+9 n^{7}+31 n^{6}+49 n^{5}+29 n^{4}-9 n^{3}-16 n^{2}-4 n}}{5 n^{5}+15 n^{4}+19 n^{3}+9 n^{2}+4}
$$

Let $d_{1}=\frac{3 n^{4}+6 n^{3}+7 n^{2}+4 n-4-2 \sqrt{n^{8}+9 n^{7}+31 n^{6}+49 n^{5}+29 n^{4}-9 n^{3}-16 n^{2}-4 n}}{5 n^{5}+15 n^{4}+19 n^{3}+9 n^{2}+4}$ and

$$
d_{2}=\frac{3 n^{4}+6 n^{3}+7 n^{2}+4 n-4+2 \sqrt{n^{8}+9 n^{7}+31 n^{6}+49 n^{5}+29 n^{4}-9 n^{3}-16 n^{2}-4 n}}{5 n^{5}+15 n^{4}+19 n^{3}+9 n^{2}+4} \text {. We next show that } d_{1}<
$$
$\min \left(\frac{1}{n+1}, f(n)\right)<d_{2}$. First observe that

$$
d_{2}-\frac{1}{n+1}=\frac{(n+1) \sqrt{n^{8}+9 n^{7}+31 n^{6}+49 n^{5}+29 n^{4}-9 n^{3}-16 n^{2}-4 n}-\left(n^{5}+3 n^{4}+3 n^{3}+4-n^{2}\right)}{\frac{1}{2}\left(5 n^{5}+15 n^{4}+19 n^{3}+9 n^{2}+4\right)(n+1)}
$$

It can be easily verified that $d_{2}>\frac{1}{n+1}$ for $n \geq 1$. Thus $d_{2} \geq \min \left(\frac{1}{n+1}, f(n)\right)$. Next observe that

$$
\frac{1}{n+1}-d_{1}=\frac{(n+1) \sqrt{n^{8}+9 n^{7}+31 n^{6}+49 n^{5}+29 n^{4}-9 n^{3}-16 n^{2}-4 n}+\left(n^{5}+3 n^{4}+3 n^{3}+4-n^{2}\right)}{\frac{1}{2}\left(5 n^{5}+15 n^{4}+19 n^{3}+9 n^{2}+4\right)(n+1)}>0
$$

thus $d_{1}<\frac{1}{n+1}$. The analytical comparison between the value of $d_{1}$ and $f(n)$ is complicated. The numerical comparison is shown in Figure 3 . Form the figure, $d_{1}$ (blue) is less than $f(n)$ for $1 \leq n \leq 100$.

Since $d_{1}<\min \left(\frac{1}{n+1}, f(n)\right)<d_{2}$, for $0 \leq \epsilon<d_{1}, \pi^{0}(n, \epsilon) \geq \hat{\pi}(n, \epsilon)$ and $k_{2}^{*}(n, \epsilon)=$ $k^{0}(n, \epsilon)=n+1$. For $d_{1} \leq \epsilon \leq \min \left(\frac{1}{n+1}, f(n)\right), \pi^{0}(n, \epsilon)<\hat{\pi}(n, \epsilon)$ and $k_{2}^{*}(n, \epsilon)=\hat{k}(n, \epsilon)=n$.

Case 2: Suppose $\frac{2}{n+1} \leq \epsilon<1$, then $k^{0}(n, \epsilon)=\hat{k}(n, \epsilon)=\frac{1}{\epsilon}$ and $\pi^{0}(n, \epsilon)=\hat{\pi}(n, \epsilon)=\epsilon$. 


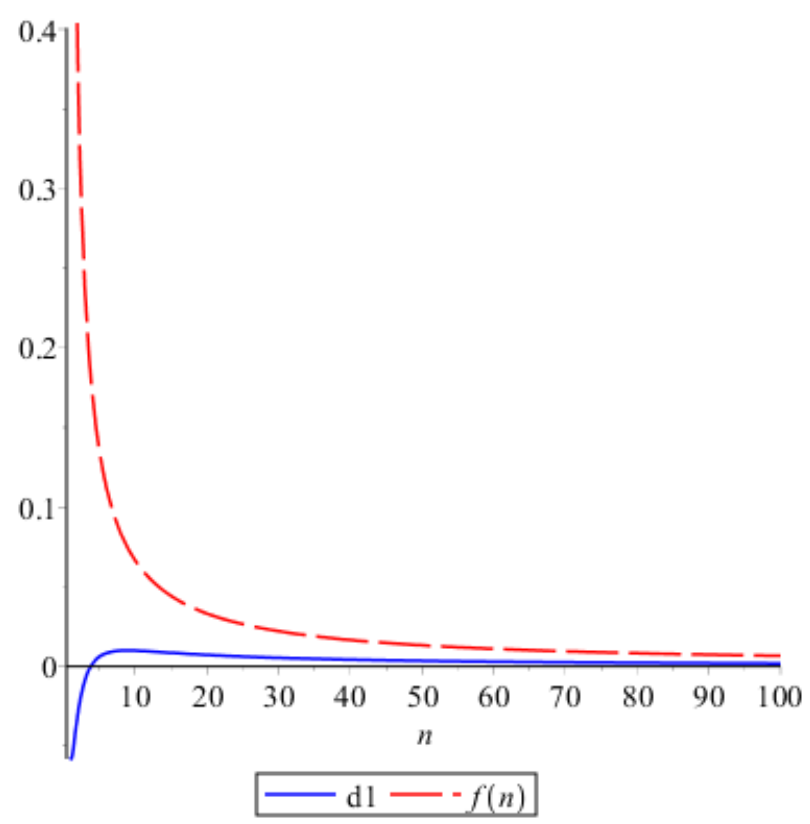

Figure 2: Comparison between $d_{1}$ and $f(n)$

Clearly $k_{2}^{*}(n, \epsilon)=\frac{1}{\epsilon}$.

Case 3: Suppose $\min \left(\frac{1}{n+1}, f(n)\right)<\epsilon<\frac{2}{n+1}$. Consider first the case $\frac{1}{n+1} \leq f(n)$. By Lemma $4 \hat{k}(n, \epsilon)<\frac{1}{\epsilon}$ thus $\hat{\pi}(n, \epsilon)>\epsilon$. Since $k^{0}(n, \epsilon)=\frac{1}{\epsilon}$ and $\pi^{0}(n, \epsilon)=\epsilon, \hat{\pi}(n, \epsilon)>\pi^{0}(n, \epsilon)$.

Consider next the case $\frac{1}{n+1}>f(n)$. (i) Suppose $\frac{1}{n+1} \leq \epsilon<\frac{2}{n+1}$, then the previous argument applies and $\hat{\pi}(n, \epsilon)>\pi^{0}(n, \epsilon)$. (ii) Suppose $f(n)<\epsilon<\frac{1}{n+1}, k^{0}(n, \epsilon)=n+1$ and $\hat{k}(n, \epsilon)=\tilde{k}(n, \epsilon)$. We next compare $\pi^{0}(n, \epsilon)$ and $\hat{\pi}(n, \epsilon)$ in this case. First observe that $\tilde{k}(n, \epsilon)<n$ for $f(n)<\epsilon<\frac{1}{n+1}$, thus $\hat{\pi}(n, \epsilon)>n\left(\pi_{1}(0, n)-\pi_{0}(1, n)\right)$. If we can show that

$$
n\left(\pi_{1}(0, n)-\pi_{0}(1, n)\right)>(n+1) \pi_{1}(n, n+1)
$$

then the proof is complete. This is indeed true since (21) holds iff $d_{1} \leq \epsilon \leq d_{2}$ and we have shown that $d_{1} \leq f(n)$ and $d_{2} \geq \frac{1}{n+1}$. Denote $g(n)=\max \left(d_{1}, 0\right)$, Proposition 3 is complete. 


\section{A.4 Proof of Proposition 5}

By Proposition 1, we focus only on the case where $k_{1}+k_{2} \leq \epsilon$. The innovator solves

$$
\max _{k_{1}, k_{2}} \overbrace{k_{1}\left(\pi_{1}\left(n-k_{1}, k_{1}+k_{2}\right)-\pi_{0}\left(n-k_{1}, k_{1}+k_{2}\right)\right)+k_{2} \pi_{1}\left(n-k_{1}, k_{1}+k_{2}\right)}^{\pi_{D}}
$$

s.t.

$$
\begin{gathered}
0 \leq k_{1} \leq n-1 \\
0 \leq k_{2} \\
k_{1}+k_{2} \leq \frac{1}{\epsilon} \\
\pi_{D}=-\frac{\left(k_{2} \epsilon^{2}+2 n \epsilon^{2}+2 \epsilon^{2}\right) k_{1}^{2}}{\left(n+k_{2}+1\right)^{2}}-\frac{\left(k_{2}^{2} \epsilon^{2}+2 k_{2} n \epsilon^{2}-n^{2} \epsilon^{2}+2 k_{2} \epsilon^{2}-2 n \epsilon^{2}-2 n \epsilon-\epsilon^{2}-2 \epsilon\right) k_{1}}{\left(n+k_{2}+1\right)^{2}}+\frac{k_{2} n^{2} \epsilon^{2}+2 k_{2} n \epsilon^{2}+2 k_{2} n \epsilon+k_{2} \epsilon^{2}+2 k_{2} \epsilon+k_{2}}{\left(n+k_{2}+1\right)^{2}}
\end{gathered}
$$

Note first that $\pi_{D}$ is continuous on $k_{1}$ and $k_{2}$. Moreover, for any $k_{2}, \pi_{D}$ is quadratic in $k_{1}$. Denote $\left(k_{1}^{*}, k_{2}^{*}\right)$ the optimal choice of the innovator. Given any $k_{2}$, let $k_{1}\left(k_{2}\right)$ be the maximizer of $\pi_{D}$. Then

$$
k_{1}\left(k_{2}\right)=\min \{\underbrace{-\frac{k_{2}^{2} \epsilon+(2 n \epsilon+2 \epsilon) k_{2}-n^{2} \epsilon-2 n \epsilon-2 n-\epsilon-2}{2 \epsilon\left(k_{2}+2 n+2\right)}}_{k_{1}^{s}}, n-1, \frac{1}{\epsilon}-k_{2}\}
$$

It can be easily verified that

$$
k_{1}^{s}<n-1 \quad \text { iff } \quad k_{2}>\underbrace{\frac{-2 n \epsilon+\sqrt{\epsilon\left(n^{2} \epsilon+2 n \epsilon+2 n+5 \epsilon+2\right)}}{\epsilon}}_{c_{1}}
$$

and

$$
k_{1}^{s}<\frac{1}{\epsilon}-k_{2} \quad \text { iff } \quad k_{2}<\underbrace{\frac{2-n \epsilon-\epsilon}{\epsilon}}_{c_{2}}
$$


It can also be verified that $c_{1} \leq c_{2}$ iff $\epsilon \leq \frac{1}{2}$. We first analyze the case $0<\epsilon \leq \frac{1}{2}$.

Case 1. $0<\epsilon<\frac{1}{2}$

Subcase 1.1: Suppose $k_{2} \leq c_{1}$, then $n-1<k_{1}^{s}<\frac{1}{\epsilon}-k_{1}$ and $k_{1}\left(k_{2}\right)=n-1$. Substituting $k_{1}$ in $\pi_{D}$ with $n-1$,

$$
\pi_{D}^{1}=-\frac{(n-1) \epsilon^{2} k_{2}^{2}+\left(2 n^{2} \epsilon^{2}-4 n \epsilon^{2}-2 n \epsilon-2 \epsilon^{2}-2 \epsilon-1\right) k_{2}+\epsilon(n-1)(n+1)(n \epsilon-3 \epsilon-2)}{\left(n+k_{2}+1\right)^{2}}
$$

It can be easily verified that $\frac{\partial \pi_{D}^{1}}{\partial k_{2}}$ is decreasing in $k_{2}$. Let $\tilde{k}_{2}$ be the solution of $\frac{\partial \pi_{D}^{1}}{\partial k_{2}}=0$. Then

$$
\tilde{k}_{2}=\frac{2 n \epsilon+n+4 \epsilon+1-2 n^{2} \epsilon}{2 n \epsilon+1}
$$

It can be verified that $\tilde{k}_{2} \leq c_{1}$ iff $\epsilon \leq \frac{1}{2}$ thus $\pi_{D}$ is maximized at $k_{2}=\tilde{k}_{2}$ for $k_{2} \leq c_{1}$.

Subcase 1.2: Suppose $c_{1} \leq k_{2} \leq c_{2}$, then $k_{1}^{s}<n-1, k_{1}^{s}<\frac{1}{\epsilon}-k_{2}$ and $k_{1}\left(k_{2}\right)=k_{1}^{s}$. Substituting $k_{1}$ in $\pi_{D}$ with $k_{1}^{s}$,

$$
\pi_{D}^{2}=\frac{k_{2}^{2} \epsilon^{2}+\left(2 n \epsilon^{2}+2 \epsilon^{2}\right) k_{2}+n^{2} \epsilon^{2}+2 n \epsilon^{2}+4 n \epsilon+\epsilon^{2}+4 \epsilon+4}{4\left(k_{2}+2 n+2\right)}
$$

It can be verified that $\pi_{D}^{2}$ is decreasing in $k_{2}$ for $0 \leq k_{2} \leq c_{2}$, thus $\pi_{D}$ is maximized at $k_{2}=c_{1}$ for $c_{1} \leq k_{2} \leq c_{2}$.

Subcase 1.3: Suppose $c_{2} \leq k_{2}$, then $\frac{1}{\epsilon}-k_{2}<k_{1}^{s}<n-1$ and $k_{1}\left(k_{2}\right)=\frac{1}{\epsilon}-k_{2}$. Since the innovator's payoff is the same for all $\left(k_{1}, k_{2}\right)$ s.t. $k_{1}+k_{2}=\frac{1}{\epsilon}$, for any $k_{2} \geq c_{2}$ the innovator obtains the same payoff. By Assumption 1 in this case $\pi_{D}$ is maximized at $k_{2}=c_{2}$ in the region $k_{2} \geq c_{2}$.

To summarize, for $k_{2} \leq c_{1}, \pi_{D}$ is maximized at $\left(k_{1}=n-1, k_{2}=\tilde{k}_{2}\right)$; for $k_{2} \in\left[c_{1}, c_{2}\right], \pi_{D}$ is maximized at $\left(k_{1}=k_{1}^{s}, k_{2}=c_{1}\right)$; for $k_{2} \geq c_{2}, \pi_{D}$ is maximized at $\left(k_{1}=\frac{1}{\epsilon}-c_{2}, k_{2}=c_{2}\right)$. Since $\pi_{D}$ is continuous in $k_{2}, \pi_{D}$ is maximized at $k_{2}=\max \left(\tilde{k}_{2}, 0\right)$. 
For $n \geq 3$, it can be easily verified that $\tilde{k}_{2} \geq 0$ iff $\epsilon \leq \frac{1}{2 n-4}$. Then

$$
k_{2}^{*}(n, \epsilon)= \begin{cases}\frac{2 n \epsilon+n+4 \epsilon+1-2 n^{2} \epsilon}{2 n \epsilon+1} & \text { if } 0<\epsilon<\frac{1}{2 n-4} \\ 0 & \text { if } \frac{1}{2 n-4} \leq \epsilon\end{cases}
$$

Next we analyze $k_{1}^{*}$. Suppose $0<\epsilon<\frac{1}{2 n-4}$. Since $k_{2}^{*}=\tilde{k}_{2}$ and $\tilde{k}_{2}<c_{1}$ (for $\left.0 \leq \epsilon<\frac{1}{2}\right)$, $k_{2}^{*}<c_{1}$. Following the analysis in subcase $1.1, k_{1}\left(k_{2}^{*}\right)=n-1$. Suppose next $\frac{1}{2 n-4} \leq \epsilon, k_{1}\left(k_{2}^{*}\right)$ then depends on the relation between $0, c_{1}$ and $c_{2}$. (i) If $0 \leq c_{1}\left(\frac{1}{2 n-4} \leq \epsilon \leq \frac{2}{3 n-5}\right)$, following the analysis of subcase $1.1, k_{1}\left(k_{2}^{*}\right)=n-1$. (ii) If $c_{1}<0 \leq c_{2}\left(\frac{2}{3 n-5} \leq \epsilon \leq \frac{2}{n+1}\right)$, following the analysis of subcase $1.2, k_{1}\left(k_{2}^{*}\right)=\left.k_{1}^{s}\right|_{k_{2}=0}=\frac{n+1}{4}+\frac{1}{2 \epsilon}$. (iii) If $c_{2}<0\left(\frac{2}{n+1}<\epsilon\right)$, following the analysis of subcase $1.3, k_{1}\left(k_{2}^{*}\right)=\frac{1}{\epsilon}$. Thus for $n \geq 3$

$$
\begin{gathered}
k_{1}^{*}(n, \epsilon)= \begin{cases}n-1 & \text { if } 0<\epsilon<\frac{2}{3 n-5} \\
\frac{n+1}{4}+\frac{1}{2 \epsilon} & \text { if } \frac{2}{3 n-5} \leq \epsilon \leq \frac{2}{n+1} \\
\frac{1}{\epsilon} & \text { if } \frac{2}{n+1}<\epsilon<\frac{1}{2}\end{cases} \\
k_{2}^{*}(n, \epsilon)= \begin{cases}\frac{2 n \epsilon+n+4 \epsilon+1-2 n^{2} \epsilon}{2 n \epsilon+1} & \text { if } 0<\epsilon<\frac{1}{2 n-4} \\
0 & \text { if } \frac{1}{2 n-4} \leq \epsilon<\frac{1}{2}\end{cases}
\end{gathered}
$$

Consider next $n=2$. It can be easily verified that $\tilde{k}_{2}>0$. Therefore $k_{2}^{*}(2, \epsilon)=\tilde{k}_{2}$ and $k_{2}^{*}(2, \epsilon)<c_{1}$ (since $\tilde{k_{2}}<c_{1}$ for $0 \leq \epsilon<\frac{1}{2}$ ). Following subcase $1.1 k_{1}^{*}(2, \epsilon)=1$. The innovator's optimal payoff is obtained for $k_{1}^{*}=1$ and $k_{2}^{*}=\left.\tilde{k}_{2}\right|_{n=2}=\frac{3}{4 \epsilon+1}$.

Case 2. $\frac{1}{2} \leq \epsilon<1$

In this case $c_{1}>c_{2}, \tilde{k_{2}}>\frac{1}{2}$ and $\tilde{k_{2}}>c_{1}$.

Subcase 2.1: Suppose $k_{2} \leq c_{2}$, then $n-1<k_{1}^{s} \leq \frac{1}{\epsilon}-k_{2}$ and $k_{1}\left(k_{2}\right)=n-1$. Following similar argument as in Subcase $1.1, \pi_{D}$ is maximized at $\min \left(\tilde{k}_{2}, c_{2}\right)$. Since $\tilde{k}_{2}>c_{1}$ and $c_{1}>c_{2}, \tilde{k}_{2}>c_{2}$. Therefore $\pi_{D}$ is maximized at $k_{1}=n-1$ and $k_{2}=c_{2}$.

Subcase 2.2: Suppose $c_{2} \leq k_{2} \leq c_{1}$, then $k_{1}^{s} \geq n-1, k_{1}^{s} \geq \frac{1}{\epsilon}-k_{2}$ and $k_{1}\left(k_{2}\right)=$ 
$\min \left(n-1, \frac{1}{\epsilon}-k_{2}\right)$. It can be easily verified that $c_{2} \leq \frac{1}{\epsilon}-n+1 \leq c_{1}$ for $\epsilon \geq \frac{1}{2}$. (i) Suppose first $c_{2} \leq k_{2} \leq \frac{1}{\epsilon}-n+1$ (or equivalently $n-1 \leq \frac{1}{\epsilon}-k_{2}$ ). Then $k_{1}\left(k_{2}\right)=n-1$. Following similar argument as in Subcase $1.1, \pi_{D}$ is $\operatorname{maximized}$ at $\min \left(\tilde{k_{2}}, \frac{1}{\epsilon}-n+1\right)$. Since $\tilde{k_{2}}>c_{1} \geq \frac{1}{\epsilon}-n+1, \pi_{D}$ is maximized at $\left(k_{1}=n-1, k_{2}=\frac{1}{\epsilon}-n+1\right)$. (ii) Suppose next $\frac{1}{\epsilon}-n+1 \leq k_{2} \leq c_{1}$ (or equivalently $n-1 \geq \frac{1}{\epsilon}-k_{2}$ ) then $k_{1}\left(k_{2}\right)=\frac{1}{\epsilon}-k_{2}$. The innovator's payoff is maximized at $k_{1}+k_{2}=\frac{1}{\epsilon}$.

Subcase 2.3: Suppose $k_{2} \geq c_{1}$, then $\frac{1}{\epsilon}-k_{2}<k_{1}^{s}<n-1$ and $k_{1}\left(k_{2}\right)=\frac{1}{\epsilon}-k_{2}$. The innovator's payoff is maximized again at $k_{1}+k_{2}=\frac{1}{\epsilon}$.

Consider first $n \geq 3$. Since $\epsilon \geq \frac{1}{2}, n-1 \geq \frac{1}{\epsilon}$ holds. Therefore Subcase 2.1 and part (i) of Subcase 2.2 are irrelevant. In this case $\pi_{D}$ is maximized at $k_{1}+k_{2}=\frac{1}{\epsilon}$. By Assumption 1. $k_{1}^{*}=\frac{1}{\epsilon}$ and $k_{2}^{*}=0$.

Consider next $n=2$. Since $\frac{1}{2} \leq \epsilon \leq 1, n-1 \leq \frac{1}{\epsilon}$ holds. Therefore Part (ii) of Subcase 2.2 and Subcase 2.3 are irrelevant. Since $\pi_{D}$ is continuous on $k_{2}$, combining Subcases 2.1 and Part (i) of Subcase $2.2 \pi_{D}$ is maximized at $\left(k_{1}=n-1, k_{2}=\frac{1}{\epsilon}-n+1\right)$. Proposition 5 follows.

\section{A.5 Proof of Proposition 6}

(i) Follows from (7) and Proposition 5 .

(ii) Let $q_{1}\left(m_{0}, m_{1}\right)$ and $q_{0}\left(m_{0}, m_{1}\right)$ be the equilibrium quantity produced by a licensee and a non-licensee, respectively, when there are $m_{0}$ firms producing at a unit cost $c$ and $m_{1}$ firms producing at a unit cost $c-\epsilon$. It can be verified that

$$
\begin{aligned}
& q_{0}\left(m_{0}, m_{1}\right)= \begin{cases}\frac{1-\epsilon m_{1}}{m_{0}+m_{1}+1} & \text { if } m_{1} \leq \frac{a-c}{\epsilon} \\
0 & \text { if } m_{1}>\frac{a-c}{\epsilon}\end{cases} \\
& q_{1}\left(m_{0}, m_{1}\right)= \begin{cases}\frac{1+\left(m_{0}+1\right) \epsilon}{m_{0}+m_{1}+1} & \text { if } m_{1} \leq \frac{a-c}{\epsilon} \\
\frac{1+\epsilon}{m_{1}+1} & \text { if } m_{1}>\frac{a-c}{\epsilon}\end{cases}
\end{aligned}
$$


Since $p_{n u}^{*}(n, \epsilon)=(c+1)-\left(\left(n-k_{1}^{*}\right) q_{0}\left(n-k_{1}^{*}, k_{1}^{*}+k_{2}^{*}\right)+\left(k_{1}^{*}+k_{2}^{*}\right) q_{1}\left(n-k_{1}^{*}, k_{1}^{*}+k_{2}^{*}\right)\right)$, by (23), (24) and Proposition 5, part (ii) of Proposition 6 follows.

\section{A.6 Proof of Proposition 7}

Lemma 5. Consider the case $n \geq 3$. (i) If $\epsilon \leq g(n), \pi_{n u}^{*}(n, \epsilon)>\pi_{u}^{*}(n, \epsilon)$. (ii) If $\frac{1}{2 n-4} \leq \epsilon<$ $\frac{2}{n+1}, \pi_{n u}^{*}(n, \epsilon)<\pi_{u}^{*}(n, \epsilon)$. (iii) If $\frac{2}{n+1} \leq \epsilon<1, \pi_{n u}^{*}(n, \epsilon)=\pi_{u}^{*}(n, \epsilon)$.

Proof. (i) If $\epsilon<g(n)$, in UA the innovator's highest payoff is $\hat{\pi}=(n+1) \pi_{1}(n, n+1)$ which is obtained when he auctions off $n+1$ licenses and all winners are entrants. In NUA if the innovator chooses $k_{1}=0$ and $k_{2}=n+1$ he obtains $\hat{\pi}$. But he can obtain more by choosing other combinations of $\left(k_{1}, k_{2}\right)$. It can be shown that $g(n)<\frac{1}{2 n-4}$ for $n \geq 3$ (the analytic proof is difficult, see Figure 3 for a numerical comparison). Thus by Proposition 5 when $\epsilon<g(n), k_{1}^{*}(n, \epsilon)>0$ and $\pi_{n u}^{*}>\hat{\pi}$.

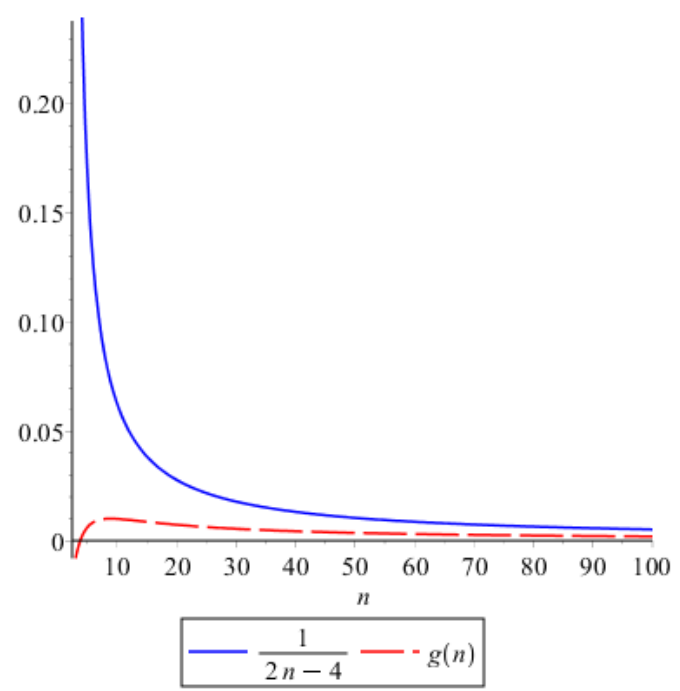

Figure 3: Comparison between $\frac{1}{2 n-4}$ and $g(n)$

(ii) If $\frac{1}{2 n-4} \leq \epsilon<\frac{2}{n+1}$ then in NUA $k_{2}^{*}(n, \epsilon)=0$. Since the advantage of NUA on UA lies only on the innovator's ability to charge for a license a higher price to entrants than to incumbent firms, this advantage disappears when $k_{2}^{*}(n, \epsilon)=0$. Moreover, for any $\left(k_{1}, k_{2}\right)$ an incumbent's willingness to pay in NUA is $\pi_{1}\left(n-k_{1}, k_{1}+k_{2}\right)-\pi_{0}\left(n-k_{1}, k_{1}+k_{2}\right)$ while it can 
be as high as $\pi_{1}\left(n-k_{1}, k_{1}+k_{2}\right)-\pi_{0}\left(n-k_{1}+1, k_{1}+k_{2}\right)$ in UA (incumbent may be willing to pay more to limit entry). Thus $\pi_{n u}^{*}(n, \epsilon) \leq \pi_{u}^{*}(n, \epsilon)$. Since $K_{n u}^{*}<\frac{1}{\epsilon}, \pi_{n u}^{*}(n, \epsilon)<\pi_{u}^{*}(n, \epsilon)$.

(iii) If $\frac{2}{n+1} \leq \epsilon<1$ the innovator auctions off in total $\frac{1}{\epsilon}$ licenses in both UA and NUA. By Proposition 1, the innovator obtains the same payoff which is the total industry profit $\epsilon$ in both auctions.

Next we focus on the analysis of $g(n)<\epsilon<\frac{1}{2 n-4}$. Clearly $\frac{1}{2 n-4}<\frac{2}{3 n-5}<\frac{2}{n+1}$ for $n \geq 3$. Thus in $G_{n u}, k_{1}^{*}(n, \epsilon)=n-1$ and $k_{2}^{*}(n, \epsilon)=\frac{2 n \epsilon+n+4 \epsilon+1-2 n^{2} \epsilon}{2 n \epsilon+1}$. In $G_{u}, \pi_{u}^{*}(n, \epsilon)$ depends on whether $\epsilon \leq f(n)$ or $\epsilon>f(n)$.

Case 1: Suppose $f(n) \leq \frac{1}{2 n-4}$ (this inequality holds for $\left.n \leq 8\right)$. Then

$$
\pi_{u}^{*}(n, \epsilon)= \begin{cases}n\left(\pi_{1}(0, n)-\pi_{0}(1, n)\right) & \text { if } g(n) \leq \epsilon \leq f(n) \\ \tilde{k}\left(\pi_{1}(n-\tilde{k}, \tilde{k})-\pi_{0}(n-\tilde{k}+1, \tilde{k})\right) & \text { if } f(n)<\epsilon<\frac{1}{2 n-4}\end{cases}
$$

We first analyze $g(n) \leq \epsilon \leq f(n)$.

$$
\pi_{n u}^{*}-\pi_{u}^{*}=\frac{\left(4 n^{5}+8 n^{4}+4 n^{3}+4 n^{2}+16 n+16\right) \epsilon^{2}}{4(n+1)^{2}(n+2)^{2}}-\frac{\left(4 n^{4}+4 n^{3}+8 n^{2}+16 n\right) \epsilon}{4(n+1)^{2}(n+2)^{2}}+\frac{n^{3}-3 n^{2}-4 n+4}{4(n+1)^{2}(n+2)^{2}}
$$

It can be easily verified that $\pi_{n u}^{*}<\pi_{u}^{*}$ iff

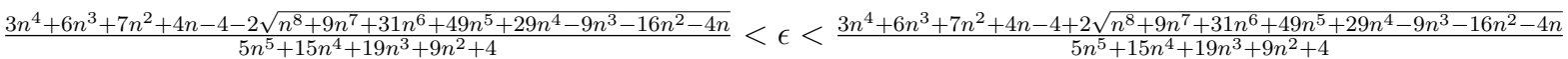

Let $e_{1}=\frac{3 n^{4}+6 n^{3}+7 n^{2}+4 n-4-2 \sqrt{n^{8}+9 n^{7}+31 n^{6}+49 n^{5}+29 n^{4}-9 n^{3}-16 n^{2}-4 n}}{5 n^{5}+15 n^{4}+19 n^{3}+9 n^{2}+4}$ and

$e_{2}=\frac{3 n^{4}+6 n^{3}+7 n^{2}+4 n-4+2 \sqrt{n^{8}+9 n^{7}+31 n^{6}+49 n^{5}+29 n^{4}-9 n^{3}-16 n^{2}-4 n}}{5 n^{5}+15 n^{4}+19 n^{3}+9 n^{2}+4}$. Figure 4 compare the value of $f(n), g(n), e_{1}$ and $e_{2}$. Note that $n \in[3,8]$ since in this section we deal with $n \geq 3$ and $f(n) \leq \frac{1}{2 n-4}$.

It can be easily verified that $g(n)$ and $e_{1}$ intersect at $g(n)=e_{1}=0$. Thus $\pi_{n u}^{*}>\pi_{u}^{*}$ for $g(n) \leq \epsilon<e_{1}$ and $\pi_{n u}^{*} \leq \pi_{u}^{*}$ for $e_{1} \leq \epsilon \leq f(n)$.

Next consider the case $f(n)<\epsilon<\frac{1}{2 n-4}$. Again, the analytic comparison between $\pi_{u}^{*}$ and 


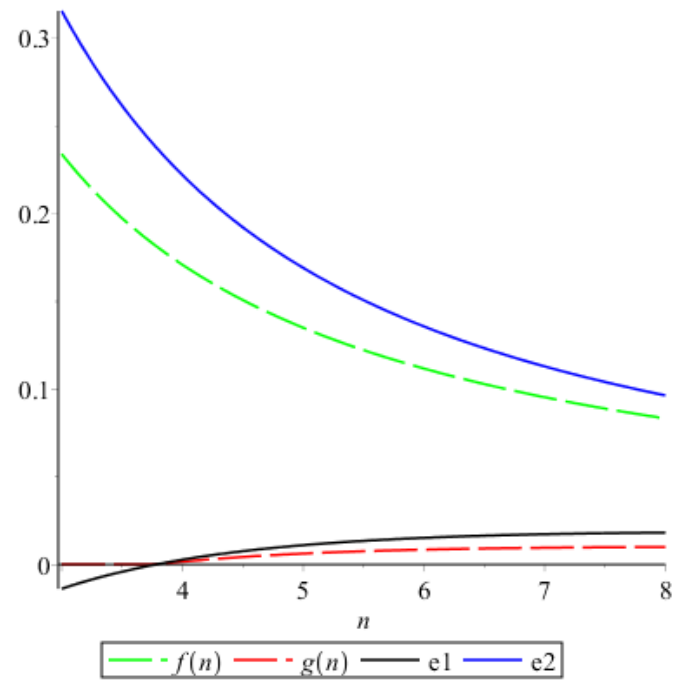

Figure 4: Comparison between $f(n), g(n), e_{1}$ and $e_{2}$

$\pi_{n u}^{*}$ is difficult and Figure 5 shows that $\pi_{u}^{*}(n, \epsilon)-\pi_{n u}^{*}(n, \epsilon) \geq 0$ numerically.

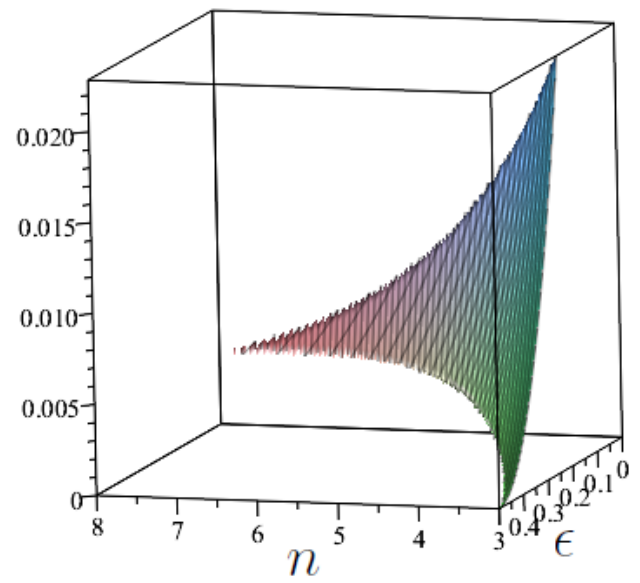

Figure 5: The value of $\pi_{u}^{*}(n, \epsilon)-\pi_{n u}^{*}(n, \epsilon)$

To summarize, in case $f(n) \leq \frac{1}{2 n-4}, \pi_{n u}^{*}>\pi_{u}^{*}$ for $g(n) \leq \epsilon<e_{1}$ and $\pi_{n u}^{*} \leq \pi_{u}^{*}$ for $e_{1} \leq \epsilon \leq \frac{1}{2 n-4}$

Case 2: Suppose $f(n)>\frac{1}{2 n-4}$ (this inequality holds for $n \geq 9$ ). Clearly for $g(n)<\epsilon<$ $\frac{1}{2 n-4}, \pi_{u}^{*}(n, \epsilon)=n\left(\pi_{1}(0, n)-\pi_{0}(1, n)\right)$. Again $\pi_{n u}^{*}<\pi_{u}^{*}$ iff $e_{1}<\epsilon<e_{2}$. Figure 6 shows that $e_{2}>\frac{1}{2 n-4}>e_{1}>g(n)$ numerically. Clearly $\pi_{n u}^{*}>\pi_{u}^{*}$ for $g(n) \leq \epsilon<e_{1}$ and $\pi_{n u}^{*} \leq \pi_{u}^{*}$ for 
$e_{1} \leq \epsilon<\frac{1}{2 n-4}$. Let $h(n)=\max \left(0, e_{1}\right)$, Proposition 7 follows.

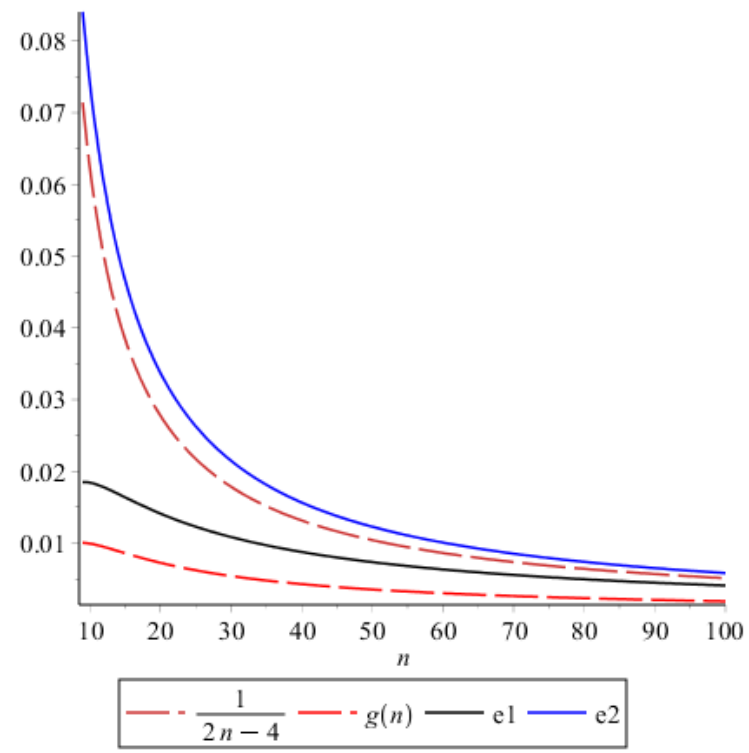

Figure 6: Comparison between $\frac{1}{2 n-4}, g(n), e_{1}$ and $e_{2}$

\section{A.7 Proof of Corollary 5}

(i) For $n \geq 3$

$$
\pi_{n u}^{*}(n, \epsilon)-\pi^{*}(n, \epsilon)= \begin{cases}\frac{(1-(2 n-4) \epsilon)^{2}}{4(n+1)} & \text { if } 0 \leq \epsilon \leq \frac{1}{2 n-4} \\ 0 & \text { if } \frac{1}{2 n-4} \leq \epsilon<1\end{cases}
$$

Let $E=\frac{(1-(2 n-4) \epsilon)^{2}}{4(n+1)}$.

$$
\frac{\partial E}{\partial \epsilon}=\frac{((2 n-4) \epsilon-1)(n-2)}{n+1}
$$

Observe that 25 is continuous in $\epsilon$ and $\frac{\partial E}{\partial \epsilon} \leq 0$ for $0<\epsilon \leq \frac{1}{2 n-4}$. Thus for any $n \geq 3$, $\pi_{n u}^{*}-\pi^{*}$ is non-increasing in $\epsilon$ for $0<\epsilon<1$.

Next observe that for $0<\epsilon<1$

$$
\pi_{n u}^{*}(n, \epsilon)-\pi^{*}(n, \epsilon)= \begin{cases}\frac{(1-(2 n-4) \epsilon)^{2}}{4(n+1)} & \text { if } 3 \leq n \leq \frac{1}{2 \epsilon}+2 \\ 0 & \text { if } \frac{1}{2 \epsilon}+2 \leq n\end{cases}
$$


and $(26)$ is continuous in $n$. Since

$$
\frac{\partial E}{\partial n}=\frac{((2 n-4) \epsilon-1)(2 n \epsilon+8 \epsilon+1)}{4(n+1)^{2}}
$$

$\pi_{n u}^{*}-\pi^{*}$ is non-increasing in $n$ for $n \geq 3$.

(ii) For $n \geq 3$

$$
p^{*}(n, \epsilon)-p_{n u}^{*}(n, \epsilon)= \begin{cases}\frac{1-(2 n-4) \epsilon}{2(n+1)} & \text { if } 0 \leq \epsilon \leq \frac{1}{2 n-4} \\ 0 & \text { if } \frac{1}{2 n-4} \leq \epsilon<1\end{cases}
$$

Clearly for any $n \geq 3, p^{*}(n, \epsilon)-p_{n u}^{*}(n, \epsilon)$ is non-increasing in $\epsilon$.

For $0<\epsilon<1$,

$$
p^{*}(n, \epsilon)-p_{n u}^{*}(n, \epsilon)= \begin{cases}\frac{1-(2 n-4) \epsilon}{2(n+1)} & \text { if } 3 \leq n \leq \frac{1}{2 \epsilon+2} \\ 0 & \text { if } \frac{1}{2 \epsilon}+2 \leq n\end{cases}
$$

Let $F=\frac{1-(2 n-4) \epsilon}{2(n+1)}$ It can be easily verified that

$$
\frac{\partial F}{\partial n}=-\frac{6 \epsilon+1}{(n+1)^{2}}
$$

Since 28 is continuous in $n$ and $\frac{\partial F}{\partial n}<0,28$ is non-increasing in $n$ for $n \geq 3$.

\section{A.8 Proof of Proposition 10}

By Proposition 9, for $n \geq 3$

$$
\pi_{0}^{*}(n, \epsilon)= \begin{cases}\frac{(n-1)\left(-(n-3) \epsilon^{2}+2 \epsilon\right)}{n+1} & \text { if } 0<\epsilon \leq \frac{2}{3 n-5} \\ \frac{(n \epsilon+\epsilon+2)^{2}}{8(n+1)} & \text { if } \frac{2}{3 n-5} \leq \epsilon \leq \frac{2}{n+1} \\ \epsilon & \text { if } \frac{2}{n+1} \leq \epsilon<1 .\end{cases}
$$


For $n \leq 2$

$$
\pi_{0}^{*}(n, \epsilon)=\frac{(n-1)\left(-(n-3) \epsilon^{2}+2 \epsilon\right)}{n+1}
$$

Consider first $n \geq 3$. First note that for any $\epsilon \geq \frac{1}{2}, \pi_{0}^{*}=\epsilon$ regardless of the value of $n$. We next focus on $0<\epsilon<\frac{1}{2}$.

Subcase 1: Suppose $n<\frac{2}{3 \epsilon}+\frac{5}{3}$ (or equivalently $0<\epsilon<\frac{2}{3 n-5}$ ). Denote

$$
\pi_{0}^{* 1}=\frac{\epsilon(n-1)(3 \epsilon+2-n \epsilon)}{n+1} .
$$

It can be easily verified that

$$
\begin{gathered}
\frac{\partial \pi_{0}^{* 1}}{\partial n}=\frac{\epsilon\left(-n^{2} \epsilon-2 n \epsilon+7 \epsilon+4\right)}{(n+1)^{2}}, \\
\frac{\partial \pi_{0}^{* 1}}{\partial n}>0 \quad \text { if } \quad 0 \leq n<2 \sqrt{2+\frac{1}{\epsilon}}-1
\end{gathered}
$$

and

$$
\frac{\partial \pi_{D}^{* 1}}{\partial n} \leq 0 \quad \text { if } \quad n \geq 2 \sqrt{2+\frac{1}{\epsilon}}-1
$$

Denote $n^{* 1}=2 \sqrt{2+\frac{1}{\epsilon}}-1$. It can be easily verified that $3<n^{* 1}<\frac{2}{3 \epsilon}+\frac{5}{3}$ for $0<\epsilon<\frac{1}{2}$. Therefore $3<n^{* 1}<\frac{2}{3 \epsilon}+\frac{5}{3}$ for $0<\epsilon \leq \frac{1}{3 n-5}$ and $n^{* 1}$ is the maximizer of $\pi_{0}^{*}$ for $3<n<\frac{2}{3 \epsilon}+\frac{5}{3}$.

Subcase 2: Suppose $\frac{2}{3 \epsilon}+\frac{5}{3} \leq n \leq \frac{2}{\epsilon}-1$ (or equivalently $\frac{2}{3 n-5} \leq \epsilon \leq \frac{2}{n+1}$ ). Deonte

$$
\pi_{0}^{* 2}=\frac{(n \epsilon+\epsilon+2)^{2}}{8(n+1)}
$$

It can be easily verified that

$$
\frac{\partial \pi_{0}^{* 2}}{\partial n}=\frac{\epsilon^{2} n^{2}+2 \epsilon^{2} n+\epsilon^{2}-4}{8(n+1)^{2}}
$$

and

$$
\frac{\partial \pi_{0}^{* 2}}{\partial n}<0 \quad \text { for } \quad 0 \leq n<\frac{2}{\epsilon}-1
$$


Therefore $n^{* 2}=\frac{2}{3 \epsilon}+\frac{5}{3}$ is the maximizer of $\pi_{0}^{* 2}$ for $\frac{2}{3 \epsilon}+\frac{5}{3} \leq n \leq \frac{2}{\epsilon}-1$.

Subcase 3: Suppose $\frac{2}{\epsilon}-1 \leq n\left(\frac{2}{n+1} \leq \epsilon \leq \frac{1}{2}\right)$. Then $\pi_{0}^{*}(n, \epsilon)=\epsilon$ and the innovator's payoff is the same for any $\frac{2}{\epsilon}-1 \leq n$.

Combining subcases $1-3$, for $n \geq 3$, since $\pi_{0}^{*}$ is continuous in $n, n^{*}=2 \sqrt{2+\frac{1}{\epsilon}}-1$ is the maximizer of $\pi_{0}^{*}$. Let $\pi_{D}^{*}$ be the innovator's equilibrium payoff when $n=n^{*}$.

$$
\pi_{D}^{*}= \begin{cases}\frac{2 \epsilon(2 \epsilon+1-\sqrt{\epsilon(2 \epsilon+1)})(\sqrt{\epsilon(2 \epsilon+1)}-\epsilon)}{\sqrt{\epsilon(2 \epsilon+1)}} & \text { if } 0<\epsilon<\frac{1}{2} \\ \epsilon & \text { if } \frac{1}{2} \leq \epsilon<1\end{cases}
$$

Consider next $n=2$. By $29,,\left.\pi_{0}^{*}\right|_{n=2}=\frac{1}{3} \epsilon^{2}+\frac{2}{3} \epsilon$. Finally consider the case $n=1$. By (29) the innovator obtains 0 since we restrict $k \leq n-1$. To provide a more reasonable comparison we assume in this case that the innovator sells the license to the incumbent firm by fixed fee. The innovator's payoff is then $\left.\pi_{0}^{*^{\prime}}\right|_{n=1}=\pi_{1}(0,1)-\pi_{1}(1,0)=\frac{1}{4} \epsilon^{2}+\frac{1}{2} \epsilon$.

Figure 7 provides the comparison of the innovator's payoff when $n^{*}=2 \sqrt{2+\frac{1}{\epsilon}}-1, n=2$ and $n=1$. Clearly in $G_{0}$ the innovator obtains the highest payoff in an oligopoly market with $n^{*}=2 \sqrt{2+\frac{1}{\epsilon}}-1$ firms.

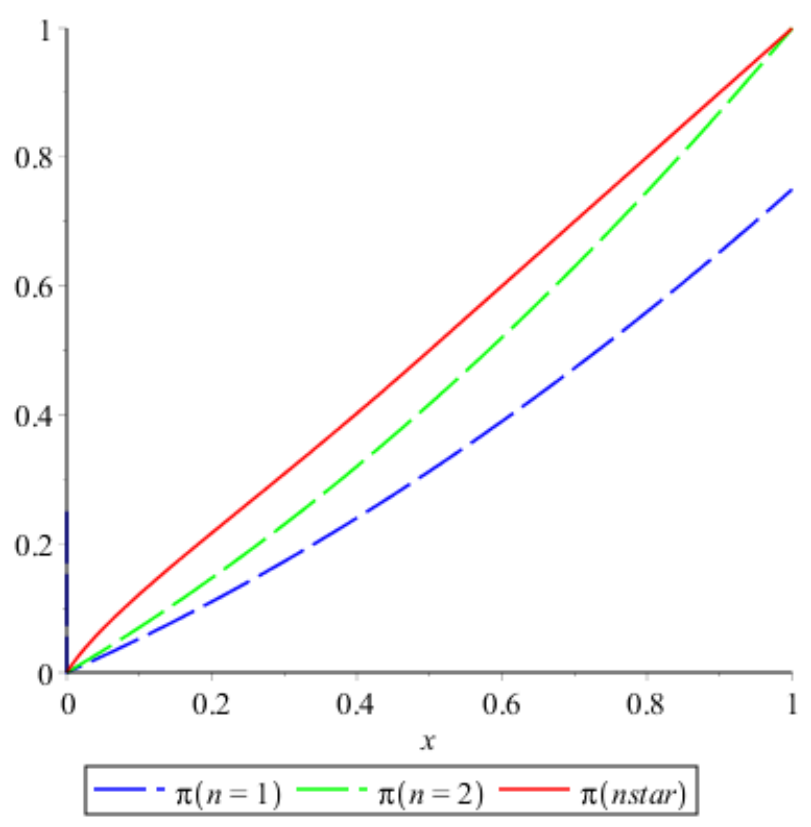

Figure 7: The innovator's payoff under different $n$ 


\section{A.9 The maximizer of $\pi_{s u}^{*}(n, \epsilon)$}

Let $\left(k_{1}^{s *}(n, \epsilon), k_{2}^{s *}(n, \epsilon)\right)$ be the maximizer of $\pi_{s u}^{*}(n, \epsilon)$.

For $n \geq 3$

$$
k_{1}^{s *}(n, \epsilon)= \begin{cases}0 & \text { if } 0<\epsilon<r(n) \\ n-1 & \text { if } r(n) \leq \epsilon \leq \frac{2}{3 n-5} \\ \frac{n+1}{4}+\frac{1}{2 \epsilon} & \text { if } \frac{2}{3 n-5} \leq \epsilon \leq \frac{2}{n+1} \\ \frac{1}{\epsilon} & \text { if } \frac{2}{n+1} \leq \epsilon<1\end{cases}
$$

and

$$
k_{2}^{s *}(n, \epsilon)= \begin{cases}n+1 & \text { if } 0<\epsilon<r(n) \\ 2 \sqrt{2+\frac{1}{\epsilon}}-(n+1) & \text { if } r(n) \leq \epsilon \leq \frac{4}{n^{2}+2 n-7} \\ 0 & \text { if } \frac{4}{n^{2}+2 n-7} \leq \epsilon<1 .\end{cases}
$$

For $n=2$

$$
k_{1}^{s *}(2, \epsilon)= \begin{cases}0 & \text { if } 0<\epsilon<r(2) \\ 1 & \text { if } r(2) \leq \epsilon \leq 1\end{cases}
$$

and

$$
k_{2}^{s *}(2, \epsilon)= \begin{cases}3 & \text { if } 0<\epsilon<r(2) \\ 2 \sqrt{2+\frac{1}{\epsilon}}-3 & \text { if } r(2) \leq \epsilon \leq \frac{1}{2} \\ \frac{1}{\epsilon}-1 & \text { if } \frac{1}{2} \leq \epsilon<1 .\end{cases}
$$

For $n=1, k_{1}^{s *}(1, \epsilon)=0$ and $k_{2}^{s *}(1, \epsilon)=28$

\section{A.10 Proof of Proposition 13}

Let $\left(0, k_{2}^{0}\right)$ and $\left(\hat{k}_{1}, \hat{k}_{2}\right)$ be maximizers of $\pi_{s u}^{0}(n, \epsilon)$ and $\hat{\pi}_{s u}(n, \epsilon)$, respectively. Clearly either $\left(0, k_{2}^{0}\right)$ or $\left(\hat{k}_{1}, \hat{k}_{2}\right)$ is a maximizer of $\pi_{s u}^{*}(n, \epsilon)$.

\footnotetext{
${ }^{8}$ In $G_{s u}$ we restrict $k_{1} \leq n-1$ therefore $k_{1}^{s *}(1, \epsilon)=0$. If, instead, an auction with minimum reservation price is conducted to the monopoly incumbent, there are parameters under which the innovator sells licenses to the incumbent firm in addition to entrants.
} 


\section{Lemma 6.}

$$
k_{2}^{0}(n, \epsilon)= \begin{cases}n+1 & \text { if } 0<\epsilon<\frac{1}{n+1} \\ \frac{1}{\epsilon} & \text { if } \frac{1}{n+1} \leq \epsilon<1\end{cases}
$$

Proof. Easy to verify.

Next we focus on the analysis of $\hat{\pi}_{s u}(n, \epsilon)$. Note that $n=1$ does not apply here since $k_{1}=0$ in this case.

Lemma 7. (i) For $n \geq 3$

$$
\begin{gathered}
\hat{k}_{1}(n, \epsilon)= \begin{cases}n-1 & \text { if } 0<\epsilon \leq \frac{2}{3 n-5} \\
\frac{n+1}{4}+\frac{1}{2 \epsilon} & \text { if } \frac{2}{3 n-5} \leq \epsilon \leq \frac{2}{n+1} \\
\frac{1}{\epsilon} & \text { if } \frac{2}{n+1} \leq \epsilon<1\end{cases} \\
\hat{k}_{2}(n, \epsilon)= \begin{cases}2 \sqrt{2+\frac{1}{\epsilon}}-(n+1) & \text { if } 0<\epsilon \leq \frac{4}{n^{2}+2 n-7} \\
0 & \text { if } \frac{4}{n^{2}+2 n-7} \leq \epsilon<1\end{cases}
\end{gathered}
$$

(ii) For $n=2$

$$
\begin{gathered}
\hat{k}_{1}(2, \epsilon)=n-1 \\
\hat{k}_{2}(2, \epsilon)= \begin{cases}2 \sqrt{2+\frac{1}{\epsilon}}-(n+1) & \text { if } 0<\epsilon \leq \frac{1}{2} \\
\frac{1}{\epsilon}-(n-1) & \text { if } \frac{1}{2} \leq \epsilon<1\end{cases}
\end{gathered}
$$

Note that $\frac{4}{n^{2}+2 n-7} \leq \frac{2}{3 n-5}$ for $n \geq 3$.

Proof. See A.11 of the Appendix.

To find the optimal licensing strategy of the innovator, we next compare $\pi_{s u}^{0}(n, \epsilon)$ and $\hat{\pi}_{s u}(n, \epsilon)$.

Lemma 8. For $n \geq 2, \pi_{s u}^{0}(n, \epsilon)>\hat{\pi}_{s u}(n, \epsilon)$ iff $\epsilon<r(n)$.

The formula of $r(n)$ is quite complicated and it appears in the Appendix A.1. 
Proof. See A.12 of the Appendix.

We are now ready to characterize the optimal licensing strategy of the innovator.

Proposition 15. For $n \geq 2$

$$
k_{1}^{*}(n, \epsilon)= \begin{cases}0 & \text { if } 0<\epsilon<r(n) \\ \hat{k}_{1}(n, \epsilon) & \text { if } r(n) \leq \epsilon<1\end{cases}
$$

and

$$
k_{2}^{*}(n, \epsilon)= \begin{cases}n+1 & \text { if } 0<\epsilon<r(n) \\ \hat{k}_{2}(n, \epsilon) & \text { if } r(n) \leq \epsilon<1 .\end{cases}
$$

Proof. Follows immediately from lemmas 6,7 and 8 .

\section{A.11 Proof of Lemma 7}

We first shows that the innovator in SUA sells licenses to entrants iff he sells licenses to all (but one) incumbent firms.

Lemma 9. For any $n \geq 2$ and $0<\epsilon<1, \hat{k}_{2}(n, \epsilon)>0$ iff $\hat{k}_{1}(n, \epsilon)=n-1$.

Proof. Denote $k=k_{1}+k_{2}$. Suppose first $k=\frac{1}{\epsilon}$. In this case each licensee (entrant or incumbent firm) pays the entire Cournot profit and by Assumption 1 the innovator sells licenses to incumbent firms and only when he exhausts all (but 1) incumbents will he sell licenses to entrants. Suppose next $1 \leq k<\frac{1}{\epsilon}$,

$$
\frac{\partial w_{l}\left(k_{1}, k-k_{1}\right)}{\partial k_{1}}=-2 \frac{\epsilon(k \epsilon-1)}{(n-k 1+k+1)^{2}}>0
$$

For any $k, 1 \leq k<\frac{1}{\epsilon}$, the license fee paid by each licensee is increasing in the number of incumbent licensees in $k$. Therefore the innovator in this case also sells licenses to incumbents first. Lemma 9 follows. 
By Lemma 9, if $k \leq n-1, k_{1}=k$ and $k_{2}=0$. If, however, $k>n-1, k_{1}=n-1$ and $k_{2}=k-(n-1)$. Therefore

$$
\hat{\pi}_{s u}(n, \epsilon)=\max \left(\max _{1 \leq k \leq n-1} k w_{l}(k, 0), \max _{k_{2}}\left(\left(n-1+k_{2}\right) w_{l}\left(n-1, k_{2}\right)\right)\right)
$$

Suppose first $n \geq 3$. It can be verified that the maximizer of $\max _{1 \leq k \leq n-1} k w_{l}(k, 0)$ is

$$
\tilde{k}_{1}(n, \epsilon)= \begin{cases}n-1 & \text { if } 0<\epsilon \leq \frac{2}{3 n-5} \\ \frac{n+1}{4}+\frac{1}{2 \epsilon} & \text { if } \frac{2}{3 n-5} \leq \epsilon \leq \frac{2}{n+1} \\ \frac{1}{\epsilon} & \text { if } \frac{2}{n+1} \leq \epsilon<1\end{cases}
$$

and the maximizer of $\left(n-1+k_{2}\right) w_{l}\left(n-1, k_{2}\right)$ is

$$
\bar{k}_{2}(n, \epsilon)= \begin{cases}2 \sqrt{2+\frac{1}{\epsilon}}-(n+1) & \text { if } 0<\epsilon \leq \frac{4}{n^{2}+2 n-7} \\ 0 & \text { if } \frac{4}{n^{2}+2 n-7} \leq \epsilon<1\end{cases}
$$

(33) States that for $\frac{4}{n^{2}+2 n-7} \leq \epsilon<1$, the innovator is best off selling 0 licenses to entrants even if he sells $n-1$ licenses to incumbent firms. By Lemma $9 \hat{k}_{1}(n, \epsilon)=\tilde{k}_{1}(n, \epsilon)$ and $\hat{k}_{2}(n, \epsilon)=0$ in this case. As for $0<\epsilon \leq \frac{4}{n^{2}+2 n-7}$, the innovator is best off selling positive number of licenses to entrants if he sells $n-1$ licenses to incumbent firms. Since $\frac{4}{n^{2}+2 n-7} \leq \frac{2}{3 n-5}$ by 32 the innovator in this case is best off selling $n-1$ licenses to incumbent firms even if $k_{2}=0$. Therefore $\hat{k}_{1}(n, \epsilon)=n-1$ and $\hat{k}_{1}(n, \epsilon)=\hat{k}_{2}(n, \epsilon)$ in this case. Part (i) of Lemma 7 follows.

Suppose next $n=2$. By Lemma $9, \hat{k}_{1}(2, \epsilon)=1$. It can be easily verified that $\hat{k}_{1}(2, \epsilon)=$ $\min \left(2 \sqrt{2+\frac{1}{\epsilon}}-3, \frac{1}{\epsilon}-1\right)$. 


\section{A.12 Proof of Lemma 8}

By Proposition 6 it is easy to verify that

$$
\pi_{s u}^{0}(n, \epsilon)= \begin{cases}\frac{(\epsilon(n+1)+1)^{2}}{4(n+1)} & \text { if } 0<\epsilon<\frac{1}{n+1} \\ \epsilon & \text { if } \frac{1}{n+1} \leq \epsilon<1\end{cases}
$$

By Proposition 7 it is easy to verify that for $n \geq 3$

$$
\hat{\pi}_{s u}(n, \epsilon)= \begin{cases}2 \epsilon(\sqrt{1+2 \epsilon}-\sqrt{\epsilon})^{2} & \text { if } 0<\epsilon \leq \frac{4}{n^{2}+2 n-7} \\ \frac{(n-1)\left(-(n-3) \epsilon^{2}+2 \epsilon\right)}{n+1} & \text { if } \frac{4}{n^{2}+2 n-7}<\epsilon \leq \frac{2}{3 n-5} \\ \frac{(n \epsilon+\epsilon+2)^{2}}{8(n+1)} & \text { if } \frac{2}{3 n-5} \leq \epsilon \leq \frac{2}{n+1} \\ \epsilon & \text { if } \frac{2}{n+1} \leq \epsilon<1\end{cases}
$$

For $n=2$

$$
\hat{\pi}_{s u}(n, \epsilon)= \begin{cases}2 \epsilon(\sqrt{1+2 \epsilon}-\sqrt{\epsilon})^{2} & \text { if } 0<\epsilon \leq \frac{1}{2} \\ \epsilon & \text { if } \frac{1}{2} \leq \epsilon<1\end{cases}
$$

Suppose $\epsilon \geq \frac{1}{n+1}$, then $\pi_{s u}^{0}(n, \epsilon)=\epsilon$ and $\hat{\pi}_{s u}(n, \epsilon) \geq \epsilon$. In this case $\hat{\pi}_{s u}(n, \epsilon) \geq \pi_{s u}^{0}(n, \epsilon)$. We next focus on the case $0<\epsilon \leq \frac{1}{n+1}$.

Case 1: Consider first $n \geq 7$. In this case $\frac{1}{n+1} \geq \frac{2}{3 n-5}$.

Subcase 1.1: Suppose $\frac{2}{3 n-5} \leq \epsilon \leq \frac{1}{n+1}$.

$$
\hat{\pi}_{s u}(n, \epsilon)-\pi_{s u}^{0}(n, \epsilon)=-\frac{(n+1)^{2} \epsilon^{2}-2}{8(n+1)}
$$

where $\hat{\pi}_{s u}(n, \epsilon) \geq \pi_{s u}^{0}(n, \epsilon)$ iff $-\frac{\sqrt{2}}{n+1} \leq \epsilon \leq \frac{\sqrt{2}}{n+1}$. Therefore $\hat{\pi}_{s u}(n, \epsilon) \geq \pi_{s u}^{0}(n, \epsilon)$ holds for $\frac{2}{3 n-5} \leq \epsilon \leq \frac{1}{n+1}$.

Subcase 1.2: Suppose $\frac{4}{n^{2}+2 n-7} \leq \epsilon \leq \frac{2}{3 n-5}$.

$$
\pi_{s u}^{0}(n, \epsilon)-\hat{\pi}_{s u}(n, \epsilon)=\frac{\left(5 n^{2}-14 n+13\right) \epsilon^{2}}{4(n+1)}+\frac{(-6 n+10) \epsilon}{4(n+1)}+(4 n+4)^{-1}
$$


Note that $\frac{5 n^{2}-14 n+13}{4(n+1)}>0$ for $n \geq 7$. It can be easily verified that $\pi_{s u}^{0}(n, \epsilon)>\hat{\pi}_{s u}(n, \epsilon)$ iff $\epsilon<\frac{3 n-5-2 \sqrt{n^{2}-4 n+3}}{5 n^{2}-14 n+13}$ or $\epsilon>\frac{3 n-5+2 \sqrt{n^{2}-4 n+3}}{5 n^{2}-14 n+13}$.

Denote $e_{1}=\frac{3 n-5-2 \sqrt{n^{2}-4 n+3}}{5 n^{2}-14 n+13}$ and $e_{2}=\frac{3 n-5+2 \sqrt{n^{2}-4 n+3}}{5 n^{2}-14 n+13}$. Figure 8 compares the value of $e_{1}, e_{2}, \frac{4}{n^{2}+2 n-7}$ and $\frac{2}{3 n-5}$ numerically. Note that $e_{1}$ and $\frac{4}{n^{2}+2 n-7}$ intersects at $n=16.19$. Thus in case $\frac{4}{n^{2}+2 n-7} \leq \epsilon \leq \frac{2}{3 n-5}$, for $7 \leq n \leq 16.19, \hat{\pi}_{s u}(n, \epsilon)>\pi_{s u}^{0}(n, \epsilon)$ holds. For $n>16.19$, $\pi_{s u}^{0}(n, \epsilon)>\hat{\pi}_{s u}(n, \epsilon)$ iff $\frac{4}{n^{2}+2 n-7} \leq \epsilon<e_{1}$.

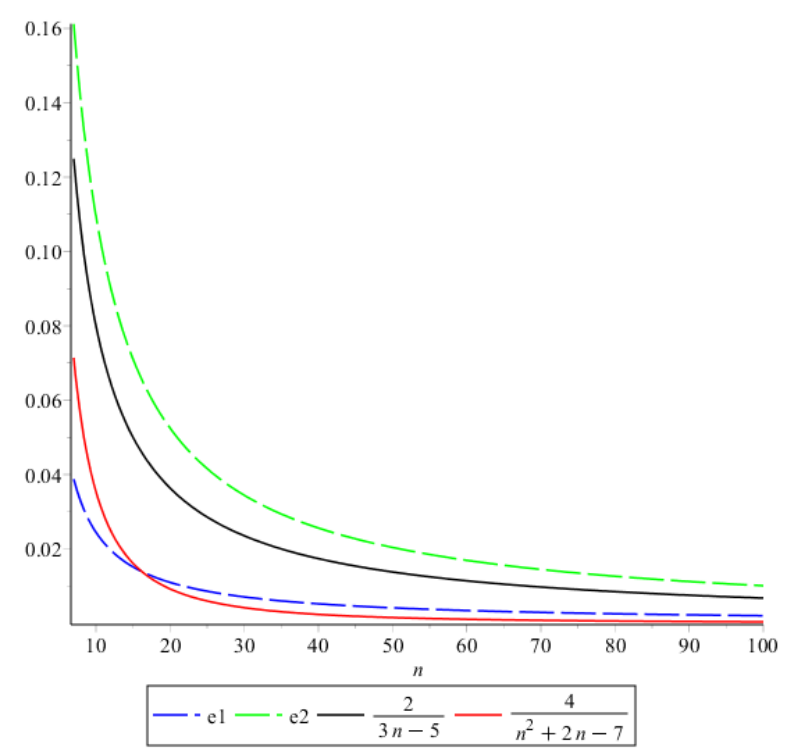

Figure 8: Comparison between $e_{1}, e_{2}, \frac{4}{n^{2}+2 n-7}$ and $\frac{2}{3 n-5}$

Subcase 1.3: Suppose $0<\epsilon \leq \frac{4}{n^{2}+2 n-7}$.

$$
\pi_{s u}^{0}(n, \epsilon)-\hat{\pi}_{s u}(n, \epsilon)=\frac{n^{2} \epsilon^{2}+\left(16 \sqrt{1+2} \epsilon \epsilon^{3 / 2}-22 \epsilon^{2}-6 \epsilon\right) n+16 \sqrt{1+2} \epsilon \epsilon^{3 / 2}-23 \epsilon^{2}-6 \epsilon+1}{4 n+4}
$$

It can be easily verified that $\pi_{s u}^{0}(n, \epsilon) \leq \hat{\pi}_{s u}(n, \epsilon)$ iff

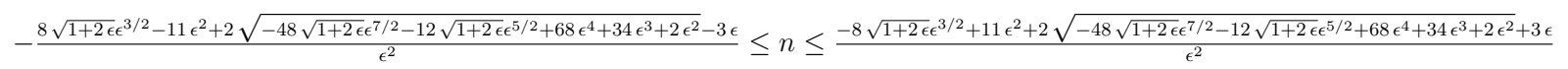

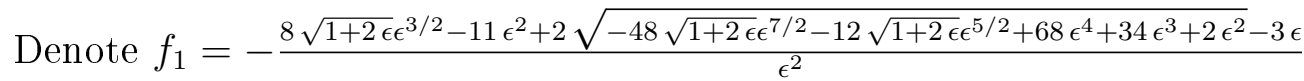

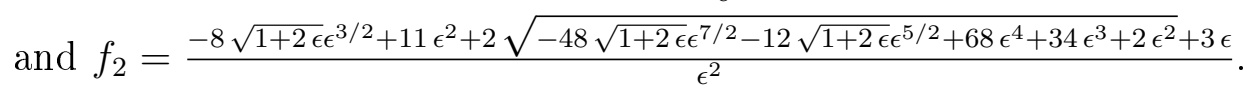

Note that for $n \geq 7,0<\epsilon \leq \frac{4}{n^{2}+2 n-7}$ iff $n \leq 2 \sqrt{2+\frac{1}{\epsilon}}-1$. Figure 9 shows that 
$f_{2}>2 \sqrt{2+\frac{1}{\epsilon}}-1$ always holds. Note that $\epsilon$ is constraint to $\frac{1}{14}$ since we are dealing in this subcase $\epsilon \leq \frac{4}{n^{2}+2 n-7}$ and $n \geq 7$.

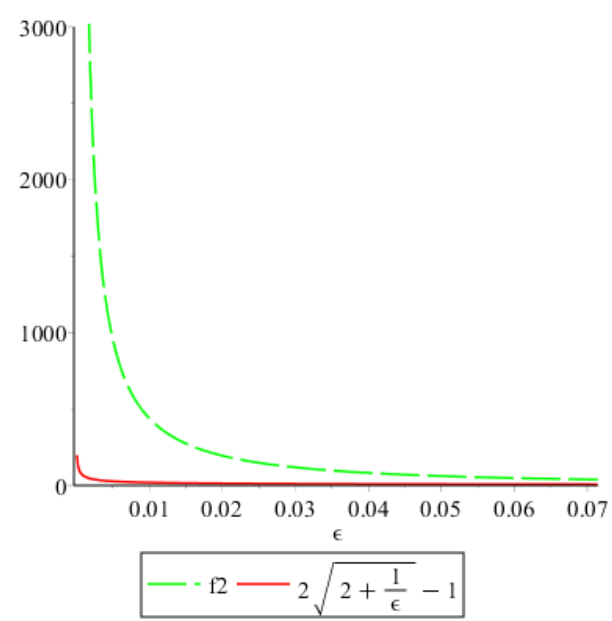

Figure 9: Comparison between $f_{2}$ and $2 \sqrt{2+\frac{1}{\epsilon}}-1$

Figure 10 compares the value of $f_{1}$ and $2 \sqrt{2+\frac{1}{\epsilon}}-1$. Note that $f_{1}$ and $2 \sqrt{2+\frac{1}{\epsilon}}-1$ intersects at $\epsilon=0.0139$ and $n=16.19$. By Figure $10, \pi_{s u}^{0}(n, \epsilon)>\hat{\pi}_{s u}(n, \epsilon)$ iff either $\epsilon<0.0139$ or $\epsilon>0.0139$ and $n<f_{1}(\epsilon)$. Or equivalently, when $0<\epsilon \leq \frac{4}{n^{2}+2 n-7}, \pi_{s u}^{0}(n, \epsilon)>$ $\hat{\pi}_{s u}(n, \epsilon)$ iff either $n>16.19$ or $n \leq 16.19$ and $\epsilon<f_{1}^{-1}(n)$ (the existence of $f_{1}^{-1}(n)$ is shown in Figure 11).

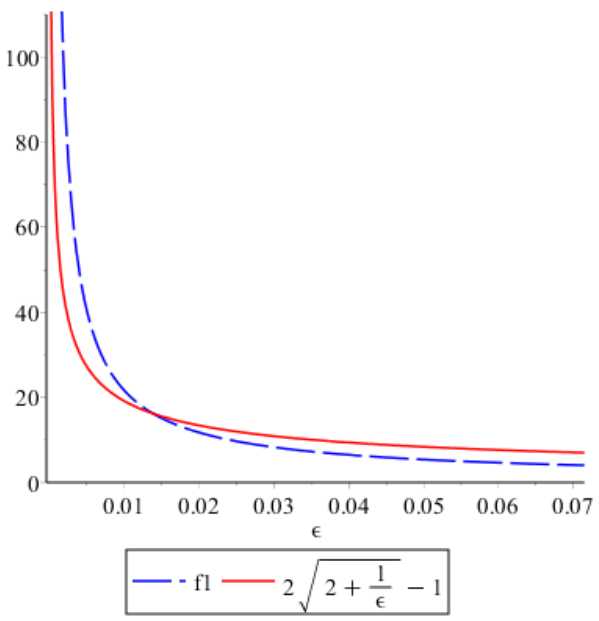

Figure 10: Comparison between $f_{1}$ and $2 \sqrt{2+\frac{1}{\epsilon}}-1$

Combining subcases 1.1-1.3, for $n \geq 17, \pi_{s u}^{0}(n, \epsilon)>\hat{\pi}_{s u}(n, \epsilon)$ iff $0<\epsilon<e_{1}$. For $7 \leq n \leq$ 
16, $\pi_{s u}^{0}(n, \epsilon)>\hat{\pi}_{s u}(n, \epsilon)$ iff $0<\epsilon<f_{1}^{-1}(n)$.

Case 2: Consider next $1+2 \sqrt{3} \leq n<7$. In this case $\frac{4}{n^{2}+2 n-7} \leq \frac{1}{n+1}<\frac{2}{3 n-5}$.

Subcase 2.1: Suppose $\frac{4}{n^{2}+2 n-7} \leq \epsilon \leq \frac{1}{n+1}$.

$$
\pi_{s u}^{0}(n, \epsilon)-\hat{\pi}_{s u}(n, \epsilon)=\frac{\left(5 n^{2}-14 n+13\right) \epsilon^{2}}{4(n+1)}+\frac{(-6 n+10) \epsilon}{4(n+1)}+(4 n+4)^{-1}
$$

where $5 n^{2}-14 n+13>0$ for $1+2 \sqrt{3} \leq n<7$. By the same argument as in Subcase $1.2, \pi_{s u}^{0}(n, \epsilon) \leq \hat{\pi}_{s u}(n, \epsilon)$ iff $e_{1} \leq \epsilon \leq e_{2}$. Figure 11 shows that $\pi_{s u}^{0}(n, \epsilon) \leq \hat{\pi}_{s u}(n, \epsilon)$ for $\frac{4}{n^{2}+2 n-7} \leq \epsilon \leq \frac{1}{n+1}$.

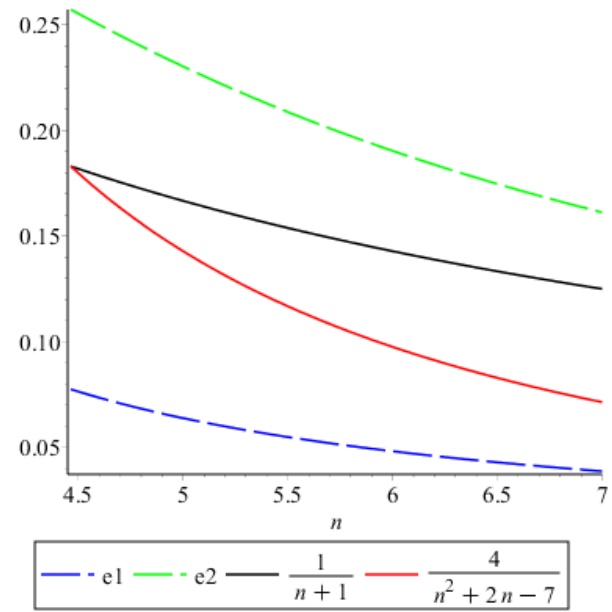

Figure 11: Comparison between $e_{1}, e_{2}, \frac{4}{n^{2}+2 n-7}$ and $\frac{1}{n+1}$

Subcase 2.2: Suppose $0<\epsilon \leq \frac{4}{n^{2}+2 n-7}$, or equivalently $1+2 \sqrt{3} \leq n \leq \min \left(7, \frac{-\epsilon+2 \sqrt{2 \epsilon^{2}+\epsilon}}{\epsilon}\right)$. Clearly $\epsilon \leq \frac{1}{2(1+\sqrt{3})}$. By the same argument as in Subcase 1.3, $\pi_{s u}^{0}(n, \epsilon) \leq \hat{\pi}_{s u}(n, \epsilon)$ iff $f_{1} \leq$ $n \leq f_{2}$. It can be easily verified that $f_{2}>7$ for $0<\epsilon \leq \frac{1}{2(1+\sqrt{3})}$. Figure 12 compares the value of $f_{1}$ and $\frac{-\epsilon+2 \sqrt{2 \epsilon^{2}+\epsilon}}{\epsilon}$. Therefore for $1+2 \sqrt{3} \leq n \leq \min \left(7, \frac{-\epsilon+2 \sqrt{2 \epsilon^{2}+\epsilon}}{\epsilon}\right), \pi_{s u}^{0}(n, \epsilon)>\hat{\pi}_{s u}(n, \epsilon)$ iff either $0<\epsilon<f_{1}^{-1}(7)$ or $f_{1}^{-1}(7) \leq \epsilon$ and $n<f_{1}$. Or equivalently, $\pi_{s u}^{0}(n, \epsilon)>\hat{\pi}_{s u}(n, \epsilon)$ iff $\epsilon<f_{1}^{-1}(n)$.

Combining subcases 2.1-2.2, for $1+2 \sqrt{3} \leq n<7, \pi_{s u}^{0}(n, \epsilon)>\hat{\pi}_{s u}(n, \epsilon)$ iff $\epsilon<f_{1}^{-1}(n)$.

Case 3: Suppose $3 \leq n \leq 1+2 \sqrt{3}$. In this case $\frac{1}{n+1} \leq \frac{4}{n^{2}+2 n-7}$. Consider $0<\epsilon \leq \frac{1}{n+1}$ (or equivalently, $3 \leq n \leq \min \left(1+2 \sqrt{3}, \frac{1}{\epsilon}-1\right)$ ). Clearly $\epsilon \leq \frac{1}{4}$. By the same argument as 


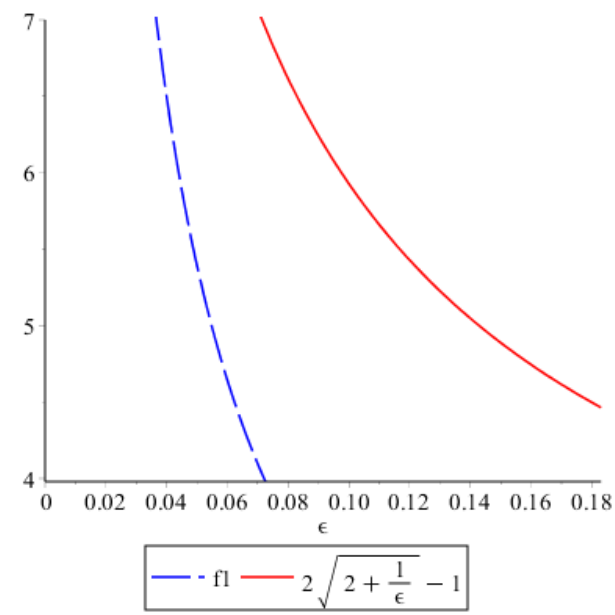

Figure 12: Comparison between $f_{1}, f_{2}$ and $\frac{-\epsilon+2 \sqrt{2 \epsilon^{2}+\epsilon}}{\epsilon}$

in Subcase 1.3, $\pi_{s u}^{0}(n, \epsilon) \leq \hat{\pi}_{s u}(n, \epsilon)$ iff $f_{1} \leq n \leq f_{2}$. Figure 13 compares the value of $f_{1}, f_{2}$ and $\frac{1}{\epsilon}-1$.

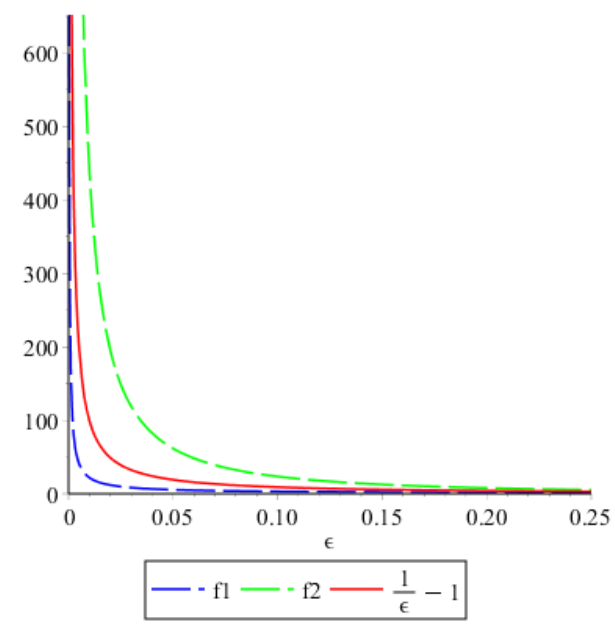

Figure 13: Comparison between $f_{1}, f_{2}$ and $\frac{1}{\epsilon}-1$

Figure 13 shows that for $3 \leq n \leq 1+2 \sqrt{3}, \pi_{s u}^{0}(n, \epsilon)>\hat{\pi}_{s u}(n, \epsilon)$ iff $\epsilon<f_{1}^{-1}(n)$.

Finally suppose $n=2$. Clearly for $\frac{1}{3} \leq \epsilon<1, \pi_{s u}^{0}(2, \epsilon) \leq \hat{\pi}_{s u}(2, \epsilon)$ since $\pi_{s u}^{0}(2, \epsilon)=\epsilon$ and $\hat{\pi}_{s u}(2, \epsilon) \geq \epsilon$. For $0<\epsilon \leq \frac{1}{3}, \pi_{s u}^{0}(2, \epsilon)>\hat{\pi}_{s u}(2, \epsilon)$ iff either $f_{1}(\epsilon)>2$ or $f_{2}(\epsilon)<2$. It can be easily verified that $f_{2}(\epsilon)>2$ for any $0<\epsilon \leq \frac{1}{3}$. Therefore $\pi_{s u}^{0}(2, \epsilon)>\hat{\pi}_{s u}(2, \epsilon)$ iff either $f_{1}(\epsilon)>2$. Or equivalently, $\pi_{s u}^{0}(2, \epsilon)>\hat{\pi}_{s u}(2, \epsilon)$ iff $\epsilon<f_{1}^{-1}(2)$.

To summarize, for any $n \geq 2, \pi_{s u}^{0}(n, \epsilon) \leq \hat{\pi}_{s u}(n, \epsilon)$ iff $0<\epsilon<r(n)$ where $r(n)=e_{1}$ for 
$n \geq 16.19$ and $r(n)=f_{1}^{-1}(n)$ for $1 \leq n<16.19$.

\section{A.13 Proof of Proposition 14}

For $n \geq 3$,

$$
\begin{aligned}
& K_{s u}^{*}(n, \epsilon)= \begin{cases}n+1 & \text { if } 0<\epsilon<r(n) \\
2 \sqrt{1+\frac{1}{\epsilon}}-2 & \text { if } r(n) \leq \epsilon \leq \frac{4}{n^{2}+2 n-7} \\
n-1 & \text { if } \frac{4}{n^{2}+2 n-7} \leq \epsilon \leq \frac{2}{3 n-5} \\
\frac{n+1}{4}+\frac{1}{2 \epsilon} & \text { if } \frac{2}{3 n-5} \leq \epsilon \leq \frac{2}{n+1} \\
\frac{1}{\epsilon} & \text { if } \frac{2}{n+1} \leq \epsilon<1\end{cases} \\
& K_{n u}^{*}(n, \epsilon)= \begin{cases}\frac{2(n+2 \epsilon)}{2 n \epsilon+1} & \text { if } 0<\epsilon \leq \frac{1}{2 n-4} \\
n-1 & \text { if } \frac{1}{2 n-4} \leq \epsilon \leq \frac{2}{3 n-5} \\
\frac{n+1}{4}+\frac{1}{2 \epsilon} & \text { if } \frac{2}{3 n-5} \leq \epsilon \leq \frac{2}{n+1} \\
\frac{1}{\epsilon} & \text { if } \frac{2}{n+1} \leq \epsilon<1\end{cases}
\end{aligned}
$$

Observe that $K_{n u}^{*}(n, \epsilon) \stackrel{\epsilon \rightarrow 0}{\longrightarrow} 2 n>n+1$. Since $K_{n u}^{*}(n, \epsilon)$ is continuous on $\epsilon$ and $K_{s u}^{*}(n, \epsilon)=$ $n+1$ for $0<\epsilon<r(n)$, Proposition 14 follows. The same argument can be applied to case $n=2$. 